\title{
The Cone Tessellation Model for Three-Dimensional Networks
}

\section{Gözde Sarışın}

Submitted to the

Institute of Graduate Studies and Research

In Partial Fulfilment of the Requirements for the Degree of

\author{
Master of Science \\ in \\ Computer Engineering
}

Eastern Mediterranean University

May 2011

Gazimağusa, North Cyprus 
Approval of the Institute of Graduate Studies and Research

\section{Prof. Dr. Elvan Yilmaz}

Director

I certify that this thesis satisfies the requirements as a thesis for the degree of Master of Science in Computer Engineering.

Assoc. Prof. Dr. Muhammad Salamah Chair, Department of Computer Engineering

We certify that we have read this thesis and that in our opinion it is fully adequate in scope and quality as a thesis for the degree of Master of Science in Computer Engineering.

Assoc. Prof. Dr. Muhammed Salamah

Supervisor

Examining Committee

1. Prof. Dr. Rza Bashirov

2. Assoc. Prof. Dr. Muhammed Salamah

3. Asst. Prof. Dr. Gürcü Öz 


\begin{abstract}
Wireless terrestrial networks are usually designed in two dimensional plane, but in real life they form three dimensional space. For navy, air force and under water applications, full connectivity and coverage are necessary to achieve good surveillance. Coverage and connectivity issues become more important to achieve full connectivity with less number of nodes. In three dimensional networks, node placement strategy is one of the most important design problems.
\end{abstract}

In this thesis, main aim is to deploy the minimum number of nodes in an effective way to get communication among nodes over a multi-hop path. Sensing range and transmission range values are main factors for our calculations. The volumetric quotient, which is the ratio of the transmission range (represented as the volume of the shape) to the sensing range (represented as the volume of the shape's circumsphere) of each node, is used as the main measure of the placement strategy. Researchers use polyhedrons to model three dimensional networks. As the volumetric quotient increases, we need less number of nodes for full coverage.

The contribution of this research is finding a cone model which gives a higher volumetric quotient than polyhedrons. The inspiration comes from satellite footprint.

The cone model was tessellated using 3Ds Max which is a modelling, animation and rendering software. Cones are deployed in special manner that has no empty space between nodes. So we can achieve full connectivity with cone model. We compared 
our results with previous researches and we achieve better results in terms of volumetric quotient and number of nodes needed compared to other models. Our result achieved the minimum of transmission ranges for both axes. Best results found in terms of maximum of minimum transmission ranges for $u$ and $v$ axes. This research shows us we can achieve full coverage with using a shape rather than polyhedrons.

Keywords: Modelling, three dimensional networks, tessellation, Wireless Sensor Networks, satellite foot-print 


\section{ÖZ}

Yeryüzündeki kablosuz ağlar genellikle 2 boyutlu olarak dizayn edilir ama gerçek hayatta 3 boyutlu bir alan oluştururlar. Askeri, hava ve su altı uygulamalarında iyi bir gözlem yapabilmek için tam kapsama ve bağlantıya ihtiyaç vardır. Az sayıda sensör kullanarak tam bağlantı sağlamada kapsama ve bağlantı önemli bir rol oynamaktadır. 3 boyutlu ağlarda en önemli dizayn problemlerinden biri sensörü yerleştirme stratejisidir.

$\mathrm{Bu}$ tezdeki asıl amaç çoklu-sıçrama yolundaki bağlantıyı sağlamak için en az sayıda sensörü en uygun şekilde yerleştirmektir. Algılama ve transfer alanı bizim hesaplamalarımızın ana faktörleridir. Volumetrik katsayı her bir sensörün transfer alanının algılama alanına bölümüyle bulunur, ki bu da yerleştirme stratejisinin ana hesaplamasıdır. Araştırmacılar 3 boyutlu ağları modellemek için çok yüzlü olan 3 boyutlu şekilleri kullanmışlardır. Volumetrik katsayı arttıkça tam kapsamayı elde etmek için daha az bağlantı noktasına ihtiyacımız olur.

Bu araştırmanın katkısı, koni modellinin çok yüzlü 3 boyutlu şekillerden daha yüksek volumetrik katsayıya sahip olmasıdır. Fikir uydu ayak izinden ortaya çıkmıştır. Koni modeli, bir modelleme, animasyon ve şekil çevirme programı olan 3Ds Max ile 3 boyutlu düzleme yerleştirilmiştir. Koniler özel bir strateji ile yerleştirilmiş olup bağlantı noktaları arasında boşluk yoktur. Böylelikle tam bağlantıyı koni modeliyle elde ettik. Sonuçlarımızı önceki araştırmalarla karşılaştırdık ve volumetrik katsayı, gerekli olan bağlantı noktası sayısı, tüm eksenler için minimum transfer alanı ve $u$ ve $w$ eksenlerinde minimum transfer alanında en iyi 
sonuçları elde ettik. Bu araştırma bize tam bağlantıyı elde etmek için çok yüzlü 3 boyutlu şekiller yerine başka şekiller kullanılabileceğini gösterdi.

Anahtar kelimeler: modelleme, 3 boyutlu ağlar, kablosuz sensör ağları, uydu ayak izi, Kelvin varsayımı, Kepler varsayımı 


\section{ACKNOWLEDGMENTS}

I would like to thank my supervisor, Assoc. Prof. Dr. Muhammed Salamah, for his continuous support and guidance through my research. This thesis would not have been completed without his encouragement and valuable ideas.

I am very grateful to Mustafa Erbilen. We discussed mathematical calculations in a long period of time, and I gained lots of information from his ideas.

I would like to thank my mum for her financial and psychological support.

At last, but not trivial, I would like to thank my precious friend Erdem, for his love, effort and patience to me. This thesis dedicated to my mum and to him. 
To My Mum and Erdem Ulutaş 


\section{TABLE OF CONTENTS}

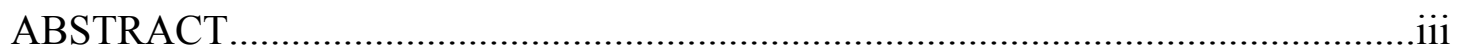

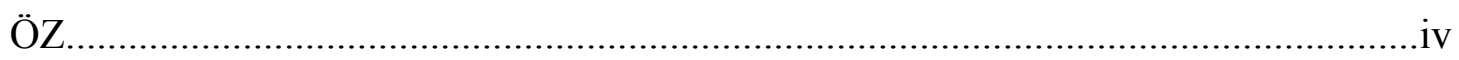

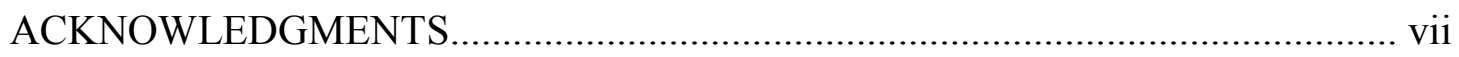

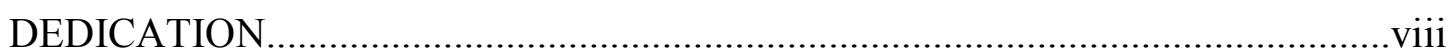

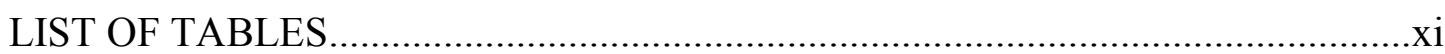

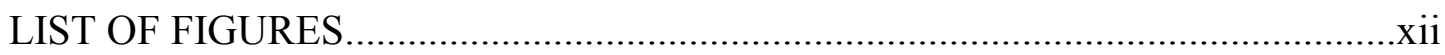

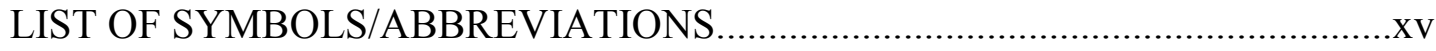

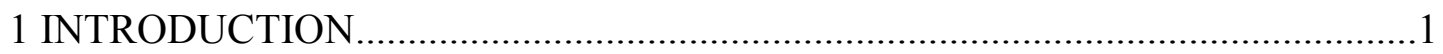

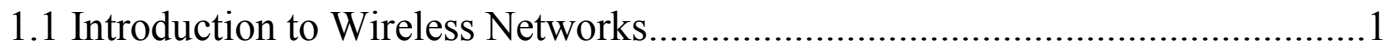

1.2 Three Dimensional Wireless Networks......................................................4

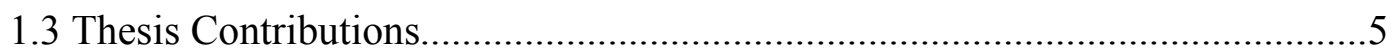

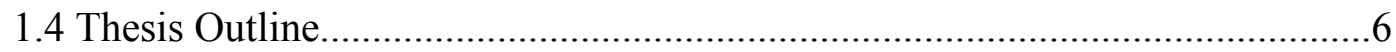

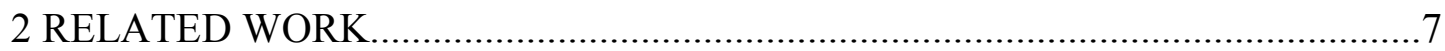

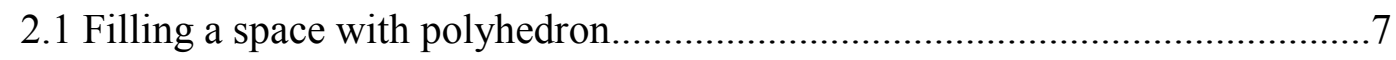

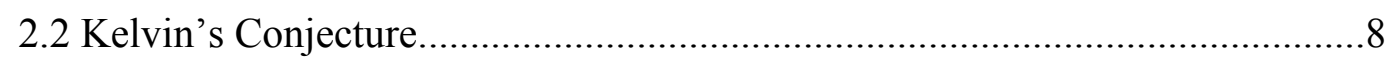

2.3 Kepler's Conjecture....................................................................... 10

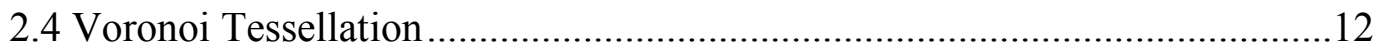

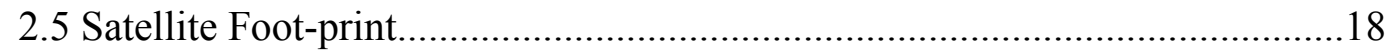

2.6 Assumptions and Goals of Related Works..............................................19

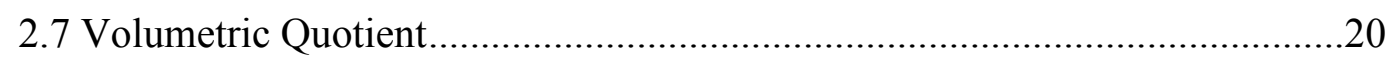

2.7.1 Volumetric Quotients for Polyhedrons ................................................23

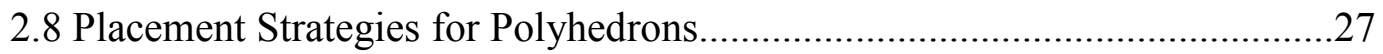


2.8.1 Placement Strategy for Cube .27

2.8.2 Placement Strategy for Hexagonal Prism..............................................27

2.8.3 Placement Strategy for Rhombic Dodecahedron.................................28

2.8.4 Placement Strategy for Truncated Octahedron...................................28

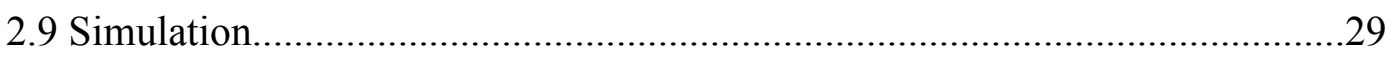

2.10 Related Works About Coverage in History....................................................32

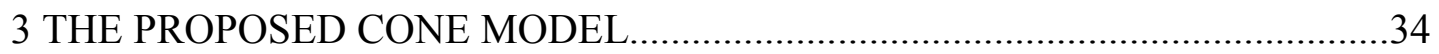

3.1 General Information About the Proposed Cone Model ...................................34

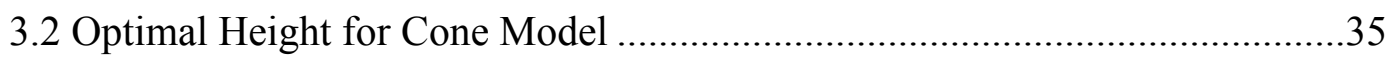

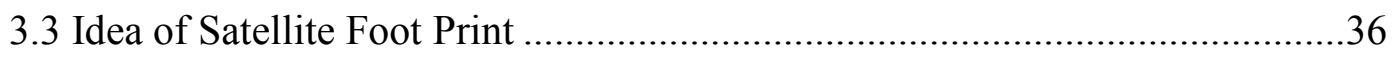

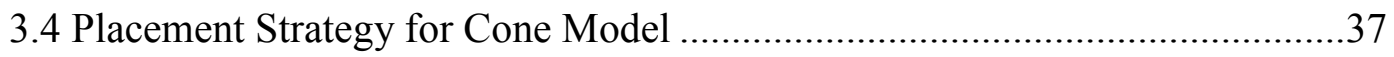

3.5 Tessellation for Cone Model in Three Dimension..........................................38

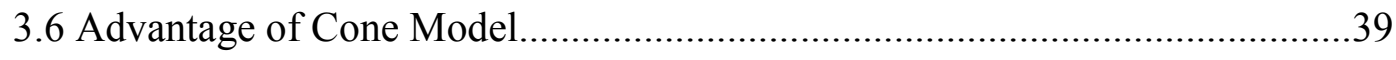

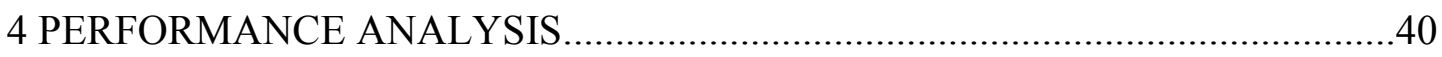

4.1 Comparison of Volumetric Quotients and Number of Nodes........................40

4.2 Comparison of Minimum Transmission Ranges for Different Models...........41

5 CONCLUSION

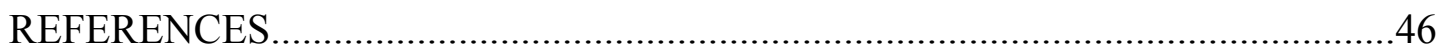

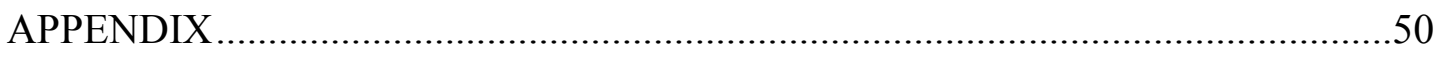

Appendix A: Snapshots of 3Ds Max for Cone Model........................................51 


\section{LIST OF TABLES}

Table 1: Volumetric quotients for different polyhedrons .26

Table 2: Minimum transmission range for previous rearch models.

Table 3: Volumetric Quotients of Models and Number of Nodes Compared to Cone......

Table 4: Comparison of Minimum Transmission Ranges for Different

Model. 


\section{LIST OF FIGURES}

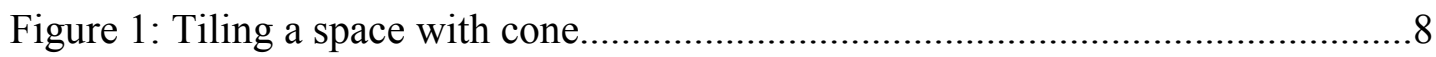

Figure 2: Shape of sphere in two dimension that is divided by cone........................10

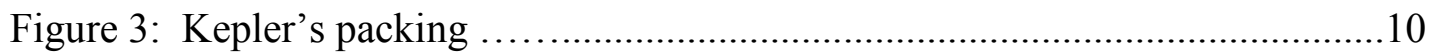

Figure 4: Diagrams of cubic close packing ABC (left) and hexagonal close packing

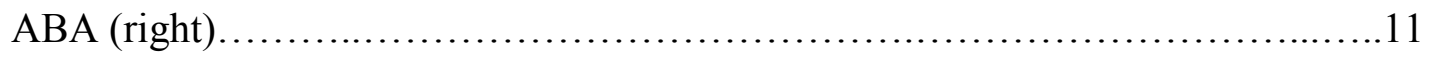

Figure 5: Two dimensional Voronoi tessellation ................................... 12

Figure 6: Node placement for cube based on CVT ….............................13

Figure 7: Hexagonal Prism node placement strategy based on CVT........................13

Figure 8: Placement of node for rhombic dodecahedron based on CVT with different radius and different size of network (a) is $15 \mathrm{mx} 15 \mathrm{mx} 15 \mathrm{~m}$ network, $\mathrm{R}=10 \mathrm{~m}$ (b) is $20 \mathrm{~m} \times 20 \mathrm{~m} \times 20 \mathrm{~m}, \mathrm{R}=5 \mathrm{~m}$

Figure 9: Placement of node for truncated octahedron based on CVT with different radius and different size of network (a) is $15 \mathrm{mx} 15 \mathrm{mx} 15 \mathrm{~m}, \mathrm{R}=10 \mathrm{~m}$ (b) is

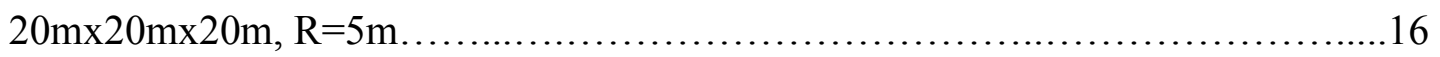

Figure 10: Honeycomb tessellation ....................................... 16

Figure 11: Cubic honeycomb ............................................. 16

Figure 12: Tessellation with rhombo- hexagonal dodecahedra .....................17

Figure 13: Two dimensional truncated octahedron tessellation...........................17

Figure 14: BCC lattice for truncated octahedron in 3 dimensional space ..............18

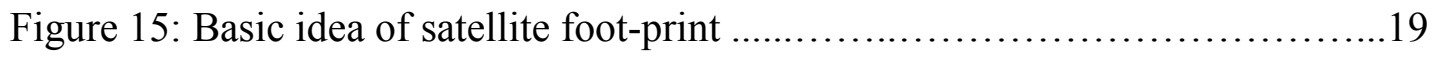

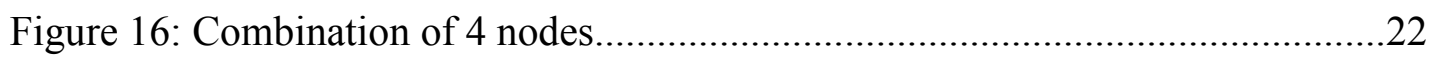

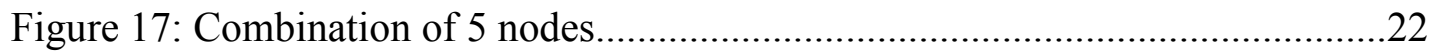


Figure 18: Combination of 6 nodes

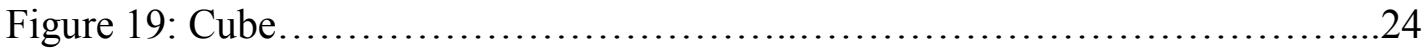

Figure 20: Hexagonal Prism ..................................................24

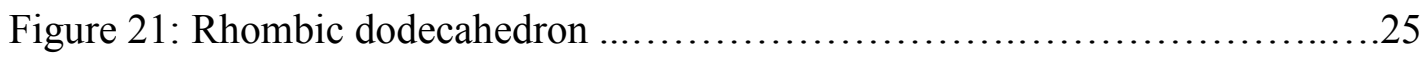

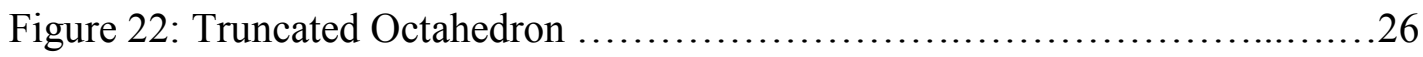

Figure 23: Tessellation for cube............................................30

Figure 24: Tessellation for hexagonal prism..................................30

Figure 25: Tessellation for rhombic dodecahedron............................... 31

Figure 26: Tessellation for truncated octahedron................................31

Figure 27: Tessellation for different network size (a) has $8 \times 8 \times 8$ nodes and (b) has

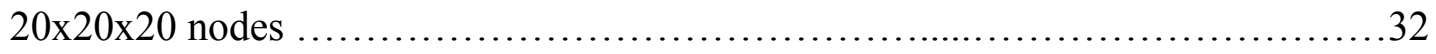

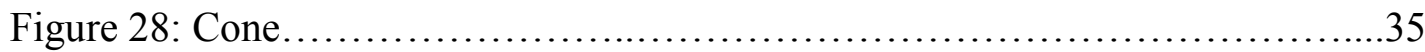

Figure 29: Octagon does not tile a plane .........................................

Figure 30: Elimination of the two duplicated nodes in the same coordinates...........36

Figure 31: Validity of distance formula...................................... 38

Figure 32: Tessellation for the cone model....................................38

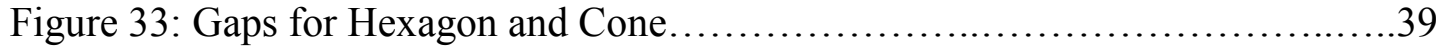

Figure A.1: Constructing a cone in three dimensional space......................51

Figure A.2: Placing the cone in three dimensional coordinate system..............51

Figure A.3: Specifying the height and the radius of cone.......................52

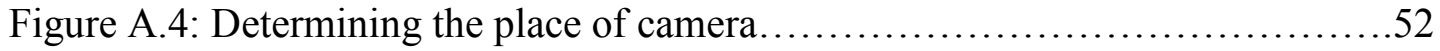

Figure A.5: Snapshot of a video file at the beginning..........................53

Figure A.6: Snapshot of a video file at 5 secs..................................53

Figure A.7: Snapshot of a video file at 15 secs.................................54

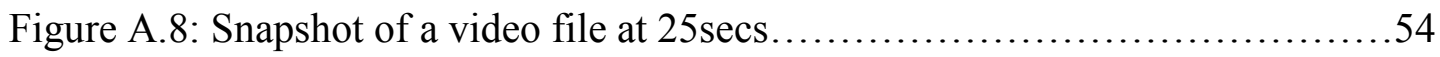


Figure A.9: Snapshot of a video file at the end...............................55

Figure A.10: Initializing video properties..................................55

Figure A.11: Render setup for completing the video..........................56

Figure A.12: Render setup for starting the video............................56

Figure A.13: Snapshot of a video while it started............................57 


\section{LIST OF SYMBOLS/ABBREVIATIONS}

CVT …............................................................... Centroidal Voronoi Tessellation

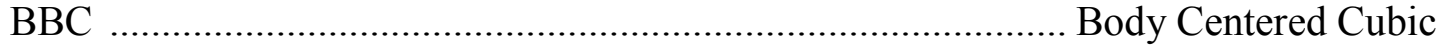




\section{Chapter 1}

\section{INTRODUCTION}

In the introduction part, coverage and connectivity problems in three dimensional wireless networks were explained. The background information about wireless networks and summary of the contributions of thesis was mentioned. Also we show the organization of this thesis.

\subsection{Introduction to Wireless Networks}

Nowadays, wireless networks have become one of the most interesting research areas for information technology. Internet gives us the latest news about our lives. But this information should be updated. This is an important issue for sensor networks because some sensors are monitoring and reporting the result to the main computer. We can give traffic monitoring as an example. People who are on a journey can easily see the road if it is crowded or not. There are many application areas for wireless sensor networks like military, civilian and health care. Military targeting system and battlefield surveillance can be done by using sensors. For civilian applications many examples can be given such as monitoring the environment, wild life and building, security and agriculture system etc. With using sensors, we can detect the animals that are becoming extinct; we can take them under control and save the natural wild life. Illness can be monitored by sensors and we can save a life. Today's sensor network is modelled like a two dimensional. That means all nodes deployed on a plane [1]. In real life nodes can be deployed anywhere in three dimension like air, ocean and ground. That is why two dimensional models are not 
valid anymore. At this present time such a scenario may not be common, for three dimensional networks many algorithms are developed in near future [2]. Nodes of an underwater sensor network can be deployed at different depths of the ocean. For example, ocean column monitoring needs the nodes to be placed at different depths of the water, because of this creating a three dimensional network is very important [3]. Additionally, underwater acoustic ad hoc and sensor networks have opened a lot of interest areas for the researchers [1], [4], [5], [6]. Weather forecasting and climate monitoring can have benefits if three-dimensional networks can be deployed in the air [2]. That means we need a good strategy for deploying the nodes in three dimensional space where we need to sense the environment. The aim of this thesis is to consider the coverage and connectivity issues of three-dimensional networks, in the sense that all nodes have the identical sensing and transmission range. Wireless sensor networks are made up with sensors. They are usually cheap and small. Also they have limited power but they have lots of functions. If data size is small, it can be delivered by the receiver without any information loss over wireless sensor networks. Also if we have mobile sensors, they can move and they can deploy and repair themselves, but these sensors are more costly than constant sensor.

However, wireless sensor networks have constraints like sensing range, transmission range and limited battery. These constraints effect life time of network, coverage and connectivity area etc. Energy conservation like energy harvesting can be used to increase network lifetime. Scheduling can save energy if we can turn off the sensor and make it sleep. Also if we can reduce the energy used in data transmission, data aggregation can also save energy. 
Most of the coverage and connectivity problems depend on transmission range and sensing range. To solve the coverage problem, sensors should be deployed very carefully. Each point of the network area must be covered by at least one sensor in the sensing range. So finding the optimal deployment strategy is very important. For maximizing the coverage area, if we can deploy sensors away from each other, the network's sensing capability will increase. There is one more advantage. If we put the nodes not very close together, we can avoid the formation of coverage holes. Coverage holes means that the area (the empty place) which is not covered by any sensor. As a solution of this problem, sensors can be deployed very close. If their transmission ranges intersect, connectivity problem can be solved.

In three dimensional networks, the coverage area of a node forms a sphere. Kelvin's Conjecture and Kepler's Conjecture have been used for finding the most efficient shape to fill the sphere. Previous researches put the nodes at the center of polyhedron shapes and they used Kelvin's Conjecture and Voronoi tessellation in three dimension. Space filling property is very important to cover a sphere and best spacefiller regular polyhedrons are dodecahedron, cube, icosahedron, tetrahedron and octahedron [2]. Most of the previous works depends on convex polyhedrons like cube, rhombic dodecahedron, hexagonal prism, and truncated octahedron to achieve full coverage and connectivity. Motivated from models like cube, hexagonal prism, rhombic dodecahedron and truncated octahedron, and by assuming the identical sensing range $\mathrm{R}$ (also transmission range is identical), for three dimensional networks we proposed a cone model to achieve better coverage and connectivity. However, we placed nodes on vertices of cones based on satellite footprint idea. 
A main objective in wireless sensor networks is to find the best deployment strategy with minimum number of nodes when $100 \%$ coverage is guaranteed [7]. In two dimensional cellular networks regular hexagon covers the circle in the most efficient way (with fewer gaps). In addition each hexagon's radius is the same as base station's maximum range. For wireless sensor networks, sensing coverage is very important. Any of the selected point in the range should be within at least one sensor's sensing range. Many algorithms [8], [9], [10], [11] developed to get 100 percent sensing coverage in two dimensional network. Network's lifetime is another important issue, to increase the lifetime energy harvesting can be used for sensor networks. Also many energy conservation protocols [12], [13], [14], [15] are developed. For a specific time period, some nodes should be activated to sense the environment. The effect of sensing coverage on performance was studied for two dimensional wireless sensor networks in [16], and only [17] for rhombic dodecahedron, and [18] for hexagonal prism studied the three dimensional cellular networks. Also in [2], they investigated the required number of nodes for truncated octahedron and maximum of the minimum transmission range. In this thesis, cone is used to model the shape of the cell, and for three dimensional space we achieved $46.35 \%$ fewer nodes than truncated octahedron model.

\subsection{Three Dimensional Wireless Networks}

Wireless networks refer to any kind of networks which is not connected by any cable. For example these kinds of networks are important for telecommunication networks and they use radio waves to communicate. However, design problem is still standing in an important place. Wireless terrestrial networks are usually designed in two dimensional plane, but in real life they form three dimensional space. In these networks, node placement strategy is the most important design problem. The main 
aim is to deploy the less number of nodes in an effective way to achieve communication between nodes over paths. The volumetric quotient that is defined as the ratio of the transmission range (represented as the volume of the shape) to the sensing range (represented as the volume of the shape's circumsphere) of each node is used as the main measure of the placement strategy. Researchers use polyhedrons to model three dimensional networks. As the volumetric quotient increases, we need less number of nodes for full coverage.

In this thesis, we proposed a cone model which gives a higher volumetric quotient than polyhedrons. The inspiration comes from satellite foot-print. For example, the required number of nodes for truncated octahedron placement strategy is $46.35 \%$ higher than the cone placement strategy. We also showed that we achieved full coverage with cone tessellation.

\subsection{Thesis Contributions}

In this thesis, a model of coverage and connectivity problems in three dimensional wireless networks with a well organized placement strategy for nodes was proposed. The thesis contributions, performance analysis, and conclusions are outlined as follows:

- Volumetric quotient approach that is the ratio of the volume of a shape to the volume of its circumsphere was used. We calculate the volumetric quotient of cone as 1.It is the highest volumetric quotient if we compare other possible space-filling polyhedrons. Keep in mind that volumetric quotient should be less than 1 for any polyhedron.

- The placement strategies for different models were shown. For each model we have three dimensional coordinate system, and nodes must be placed 
inside of this three dimensional system. There is a relation between new coordinate system and original coordinate system. Relation between them are calculated in terms of sensing range $R$ and the location of each node is defined identically using terms $c x, c y$ and $c z$. We have just small calculations to put the node to its place that is why computing the exact place is not very hard[2]. At the end, based on our calculations, cone placement strategy needs the ratio of transmission range to the sensing range $R$ must be at least 2.2360 in order to keep up connectivity between nodes.

\subsection{Thesis Outline}

The remaining part of this thesis is organized as follows: Chapter 2 represents related works of the proposed research. Chapter 3 presents the proposed analytical solution. Chapter 4 compares the performance analysis of models. Chapter 5 concludes the report and shows our reference which are very helpful for the thesis. 


\section{Chapter 2}

\section{RELATED WORK}

This Chapter presents the coverage problem in three dimensional sensor networks and it summarized the preliminaries. Section 2.1 explains the space-filling polyhedron. Kelvin's conjecture was defined in Section 2.2. Also in Section 2.3 Kepler's conjecture was discussed. In Section 2.4, voronoi tessellation was explained which is useful for previous models but it is not appropriate for our research. Because understanding the idea of voronoi tessellation is important for better understanding of our new cone model. In Section 2.5 Satellite foot print idea was described. Assumptions and goals of the previous researches are given in Section 2.6. Volumetric quotient idea was explained in Section 2.7. Placement strategies for specific polyhedrons were shown in Section 2.8. Simulation is shown in Section 2.9. Finally, there is an explanation about history of two and three dimensional networks in Section 2.10.

\subsection{Filling a Space with Polyhedron}

Combination of multi polygonal faces makes a shape in three dimensions. This shape is called polyhedron. The straight line segments where the faces meet are called edges, and the point where these edges meet are called vertices of polyhedron [2]. A polyhedron can easily fill a surrounded volume in three dimensions. Such as, prisms, pyramids and cube are polyhedrons. Since pyramids look like cones, we worked with the formula of volumetric quotient which is for polyhedrons. 
For tessellation and tiling a space-filling polyhedron is suitable for occupying a volume without any empty space. Five polyhedrons which have regular faces can have the space-filling property. These are hexagonal prism, triangular prism, cube, truncated octahedron and gyrobifastiguim. Also a combination of octahedrons and tetrahedrons can fill a space. Because the sensing area of a node is spherical and spheres are not suitable for tessellation in three dimensions, this thesis aim is to discover a shape which is different from polyhedron that best approximates the sphere. In a nutshell, the aim of this thesis is to discover a shape in the sense that if every cell can be modelled with that shape, in that case the total number of cells needed to fill a volume is relatively minimized, such that the distance from the center of a cell to its vertex is less than the sensing range $R$.

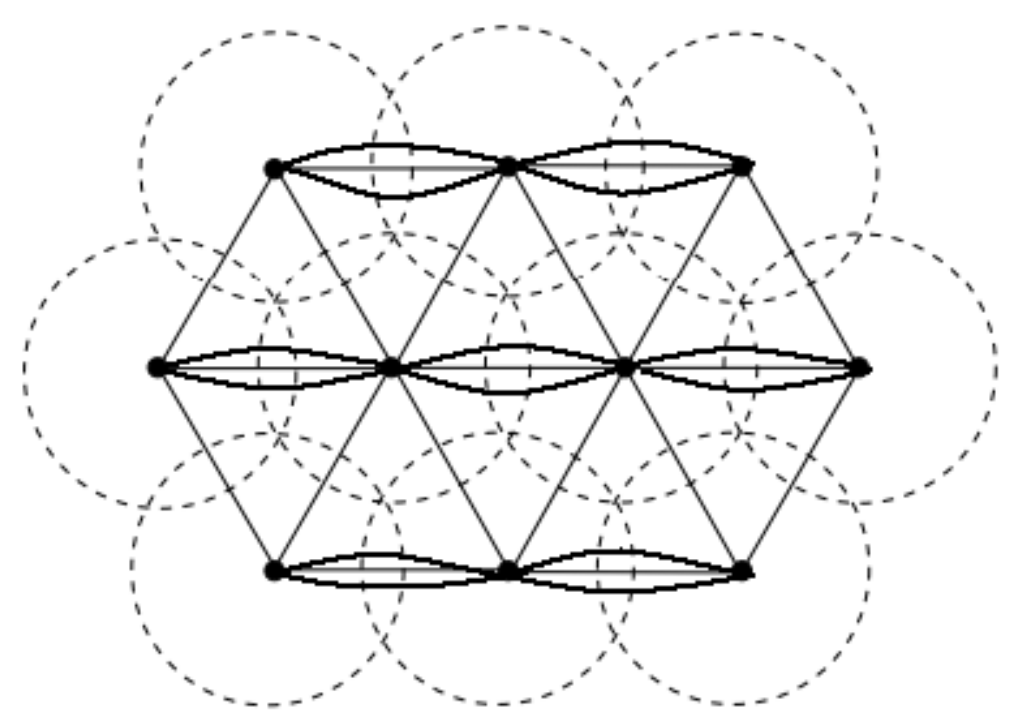

Figure 1: Tiling a space with cones

\subsection{Kelvin's Conjecture}

In 1887, Lord Kelvin tried to find the most efficient way to cover a three dimensional space with cells with same volume. His aim was minimizing the surface area (interface area). The problem was finding the highest isoperimetric quotient with the 
best space filling shape. If the volume is $V$ and surface area of a shape is $\mathrm{S}$, then its isoperimetric quotient can be defined as $36 \pi V^{2} / S^{3}$ in three dimensions [2]. The highest isoperimetric quotient belongs to sphere (it is equal to 1). Kelvin's solution was 14-sided truncated octahedron and its isoperimetric quotient was 0.765 . But he could not prove his cell is optimal. In 1994, two physicists found a different space filling structure. This structure has six 14-sided polyhedrons and two 12-sided polyhedrons with asymmetrical faces of identical volume and it isoperimetric quotient is 0.773 which is approximately $1.0 \%$ more than Kevin's cell. Still there is not any proof for these two structures.

Isoperimetric quotient can be calculated for only polyhedral multi-sided shapes. Therefore, for our work we could not calculate isoperimetric quotient but we can use the idea of volumetric quotient.

If we summarize Kelvin's conjecture, he tried to achieve minimum number from the ratio of surface area and volume from a space filling polyhedron. If polyhedron has minimum ratio, it means it is the shape that best suits the sphere. For a specific volume, sphere has the smallest surface area and for a specific surface area, sphere has the largest volume [2]. So our solution is achieving volumetric quotient that is the same with sphere using non polyhedral shape. First we thought in two dimension and expanded it in three dimension. Finally we found alternative solution to Kelvin's and Kepler's solution. 


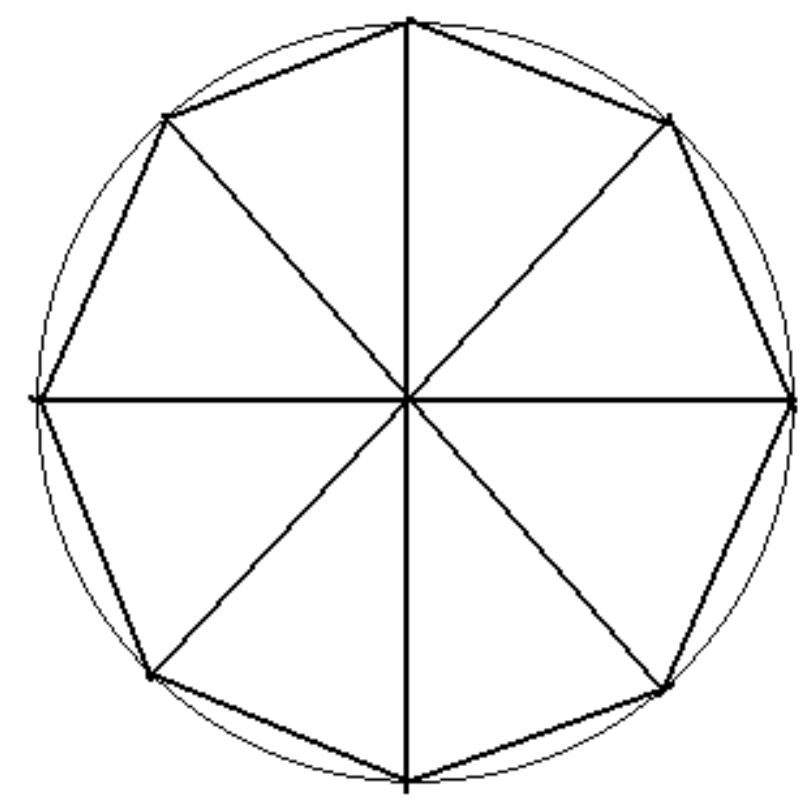

Figure 2: Shape of sphere in two dimension that is divided by cone

\subsection{Kepler's Conjecture}

Kepler's conjecture is a very similar problem to Kelvin's conjecture. In addition, Kepler's conjecture is sphere packing problem. He tried to find the optimal way to group same sized spheres.
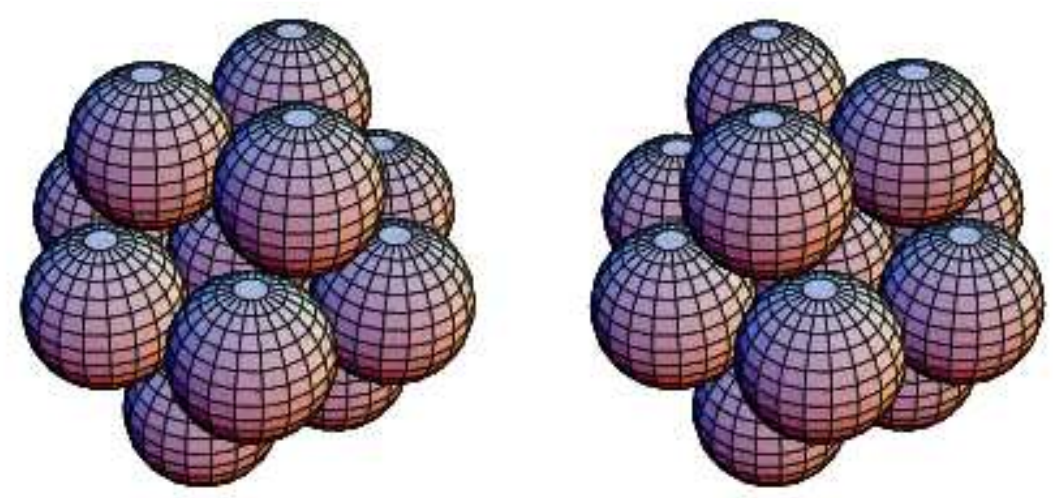

Figure 3: Kepler's packing [19]

In 1661, Kepler thought that face centered cubic lattice or hexagonal lattice (their densities are same and it is $\pi / 3 \sqrt{2}=74.048 \%$ ) was the optimal of all organizations. It was not able to proof it. 
C

(2)

B

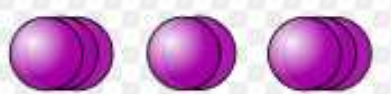

A
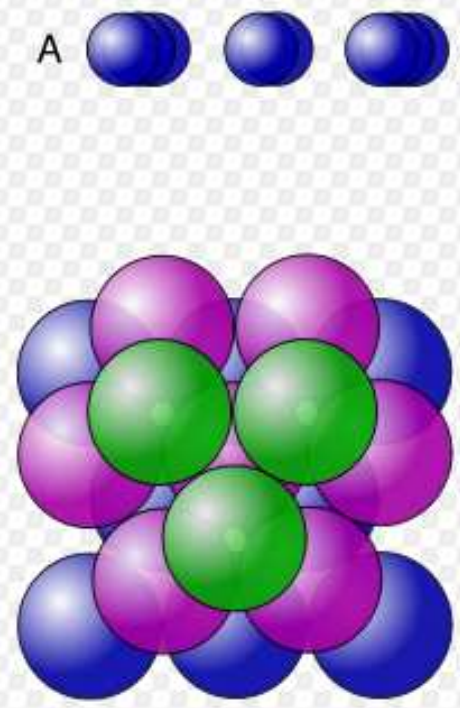

A

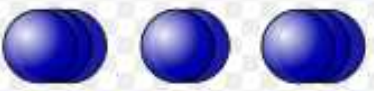

B

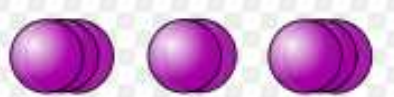

A
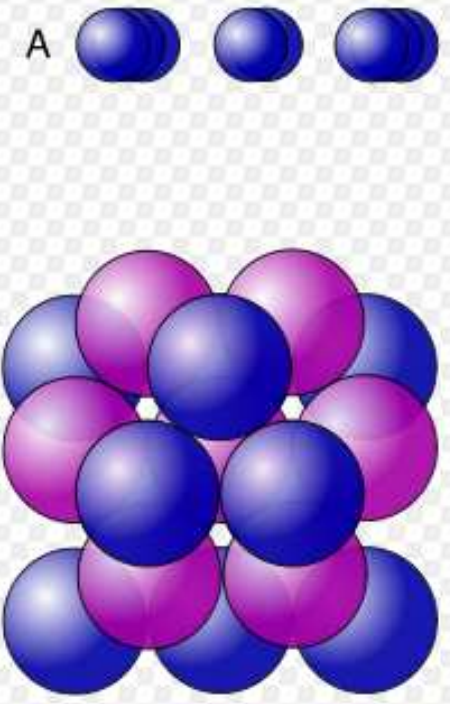

Figure 4: Diagrams of cubic close packing ABC (left) and hexagonal close packing ABA (right) [20]

Kepler's conjecture was correctly proved in 1998 after four hundred years trial and error. Most of the proofs were derived from the methods of theory in global optimization, interval arithmetic, and linear programming. The computer files include the computer code and data files for combinatorics, interval arithmetic, and for storage linear programs need more than 3 gigabytes of space [21]. Hales' proof found very hard to confirm in 1997. In 2003, the Annals of Mathematics publication started to publish some parts of this work but it was impossible to check its correctness, but some reviewers worked on confirming the proof for approximately five years and that the reviewers were sure $99 \%$ since 2003 . But, there is a publication related to this subject in 2005 , and it has not any information about the proof $[21]$. 
Face centered cubic lattice is optimal solution for sphere grouping, but this thesis is explained that cone model needs $46.35 \%$ fewer nodes for our problem using satellite foot-print idea. This result is very important and not negligible.

\subsection{Voronoi Tessellation}

Voronoi cells are very important for the placement of nodes in three dimensional networks. It is also known as Theissen or Dirichlet tessellation. Dirichlet used two dimensional and three dimensional Voronoi diagrams in his studies. Voronoi tessellation have many applications in computer science, chemistry etc. Voronoi tessellation divides the domain in a set of polygonal regions and to implement Voronoi tessellation in computer science Javascripts are very helpful. Voronoi nodes have equal distance to three or more sides to the polyhedron.

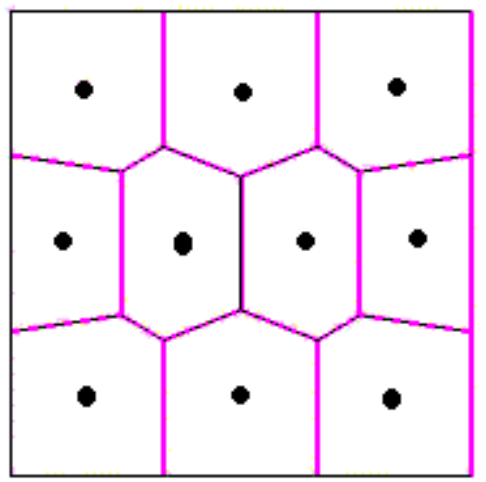

Figure 5: Two dimensional Voronoi Tessellation [22]

Definition: "In three-dimension, for any (topologically) discrete set S of points in Euclidean space, the set of all points closer to a point $c$ of $S$ than to any other point of $S$ is the interior of a convex polyhedron called the Voronoi cell of $c$. The set of such polyhedrons tessellate the whole space, and is called the Voronoi tessellation corresponding to the set S”. [2]

Previous researches take into account the above definition and they used Voronoi tessellation for finding the optimal shape of cell and optimal location of the nodes. 
Centroidal Voronoi Tessellation (CVT) is center of the shape with respect to given density function and it is used for space filling models.

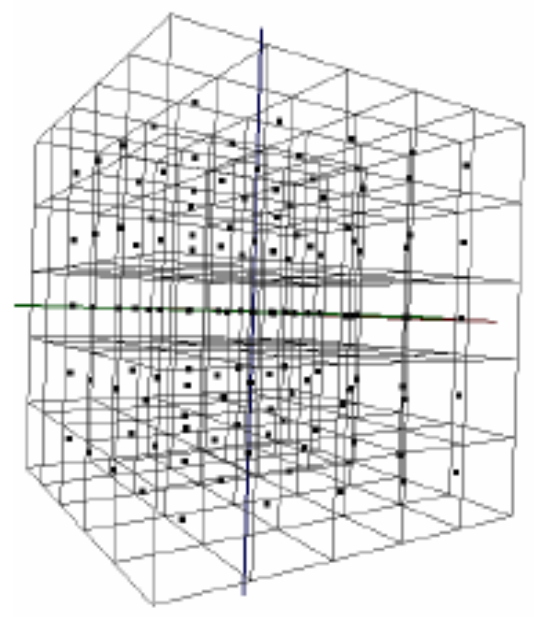

Figure 6: Node placement for cube based on CVT [2]

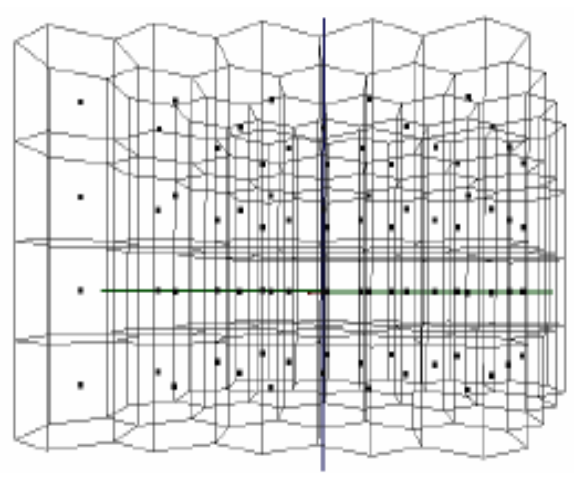

Figure 7: Hexagonal Prism node placement strategy based on CVT [2] 


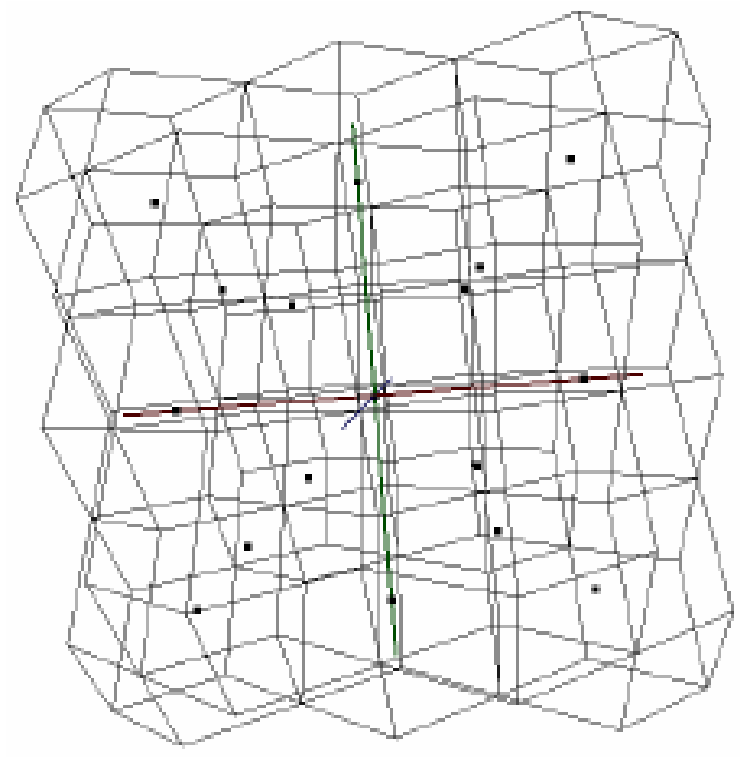

(a)

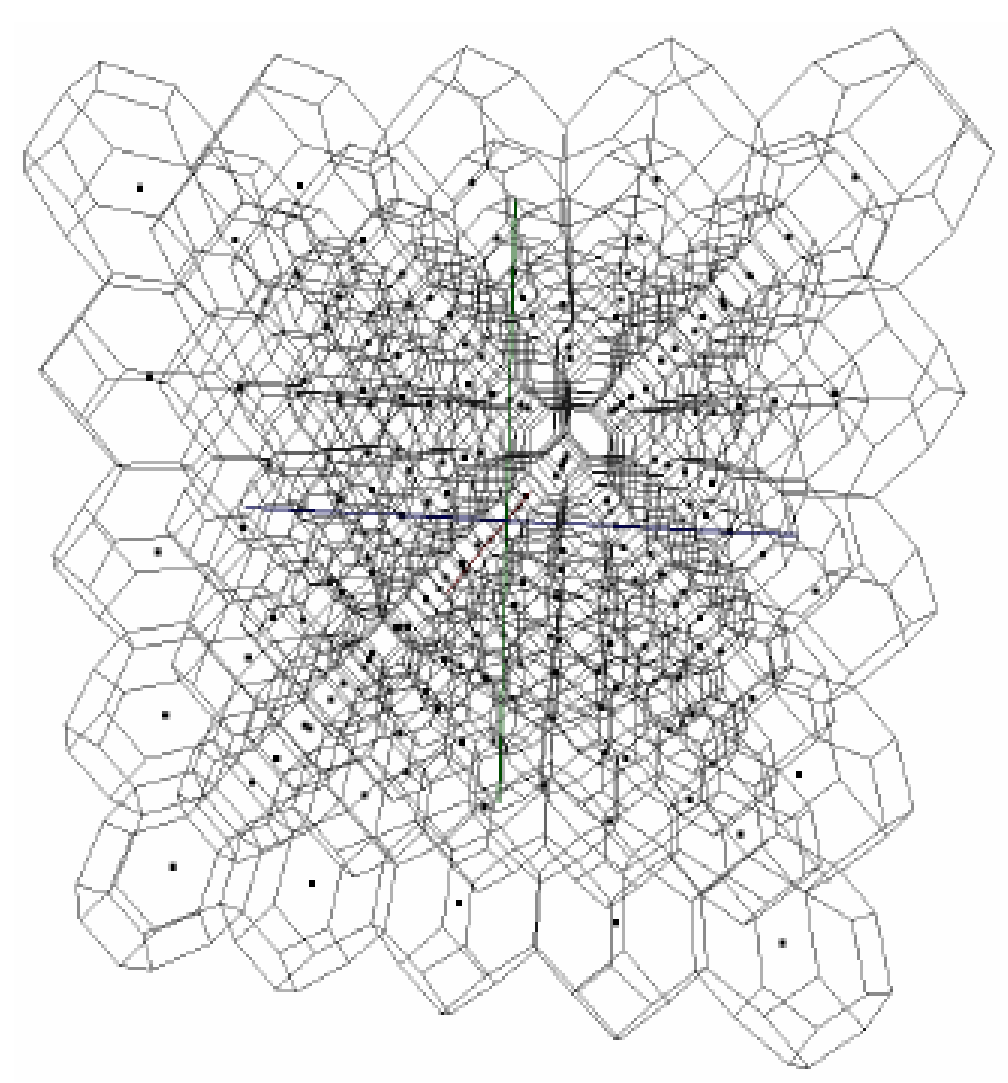

(b) 
Figure 8: Placement of node for rhombic dodecahedron based on CVT with different radius and different size of network (a) is $15 \mathrm{mx} 15 \mathrm{mx} 15 \mathrm{~m}, \mathrm{R}=10 \mathrm{~m}$ (b) is $20 \mathrm{~m} \times 20 \mathrm{~m} \times 20 \mathrm{~m}, \mathrm{R}=5 \mathrm{~m}[2]$

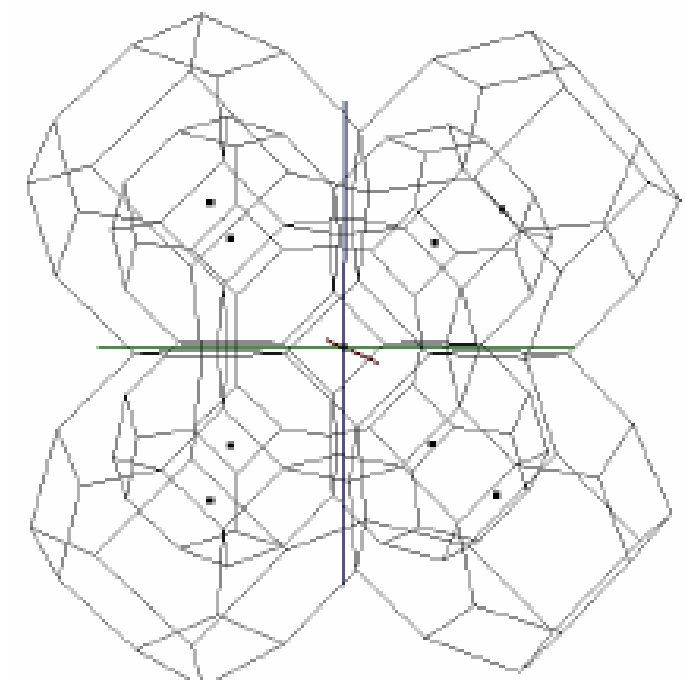

(a)

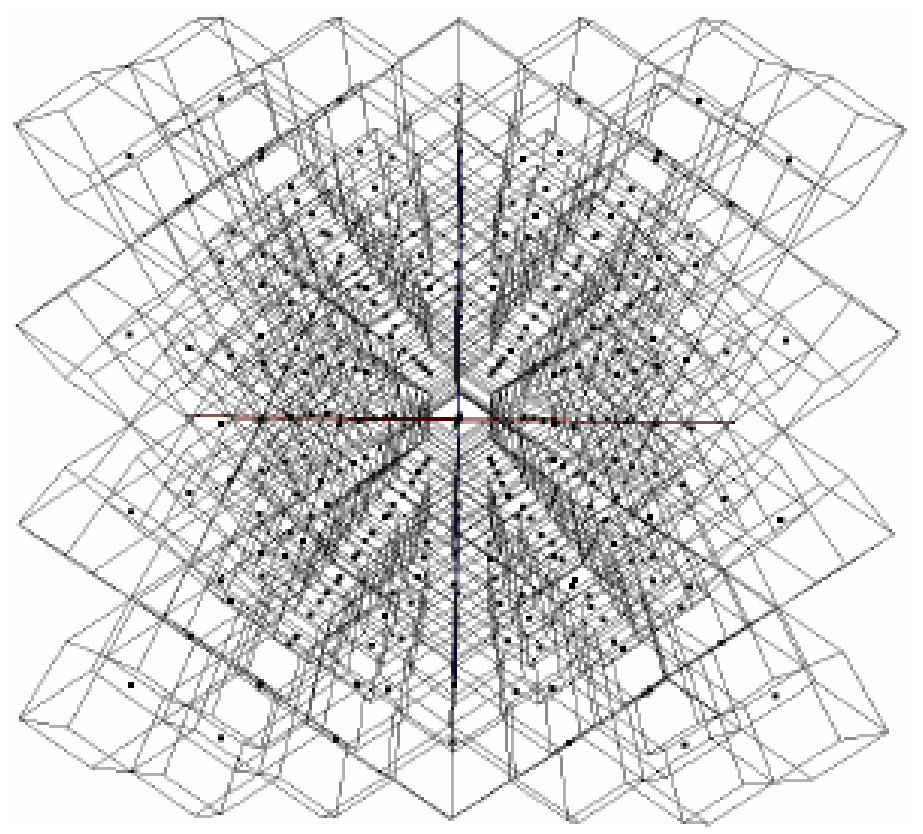

(b) 
Figure 9: Placement of node for truncated octahedron based on CVT with different radius and different size of network (a) is $15 \mathrm{mx} 15 \mathrm{mx} 15 \mathrm{~m}, \mathrm{R}=10 \mathrm{~m}$ (b) is

$$
20 \mathrm{~m} \times 20 \mathrm{~m} \times 20 \mathrm{~m}, \mathrm{R}=5 \mathrm{~m}[2]
$$

Also there are examples for two and three dimensions with regular lattices of points. A two dimensional lattice makes a tessellation like an irregular honeycomb.

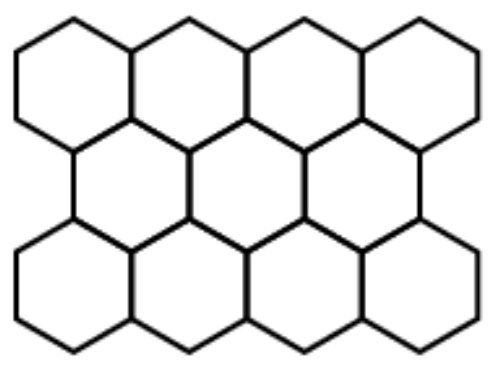

Figure 10: Honeycomb tessellation [23]

For triangular lattice and square lattice, tessellation is regular. A three dimensional cubic lattice makes a tessellation like a cubic honeycomb.

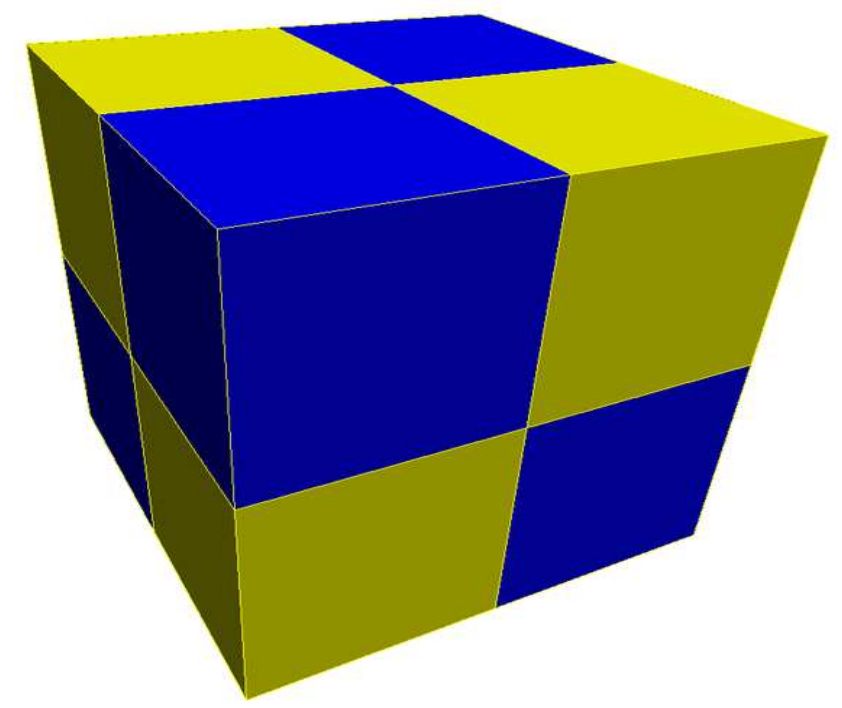

Figure 11: Cubic honeycomb [24]

Certain body centered tetragonal lattices makes a tessellation with rhombohexagonal dodecahedra. 


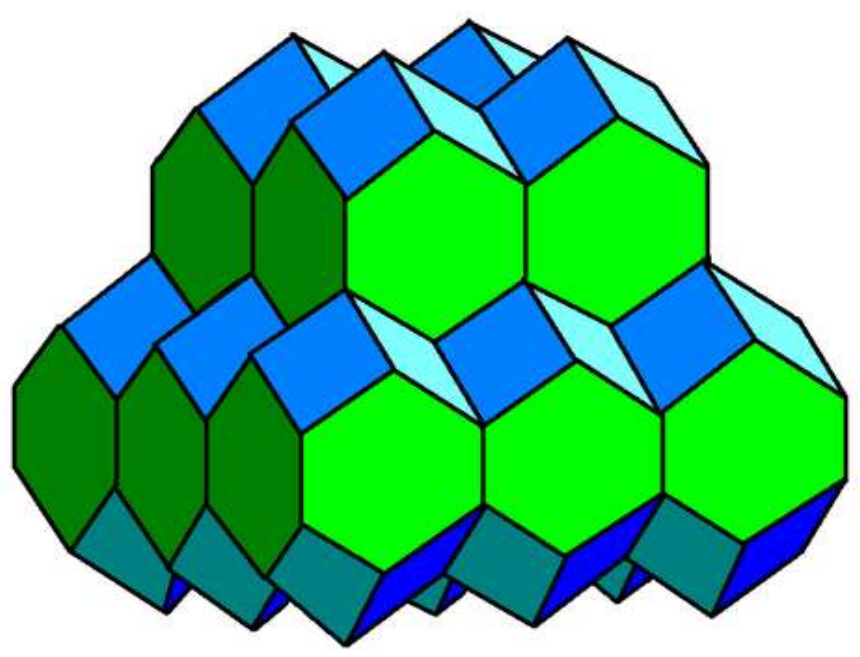

Figure 12: Tessellation with rhombo-hexagonal dodecahedra [25]

In the case of face centered cubic lattice makes a tessellation with rhombic dodecahedron. Also body centered cubic lattice constructs a tessellation with truncated octahedron.

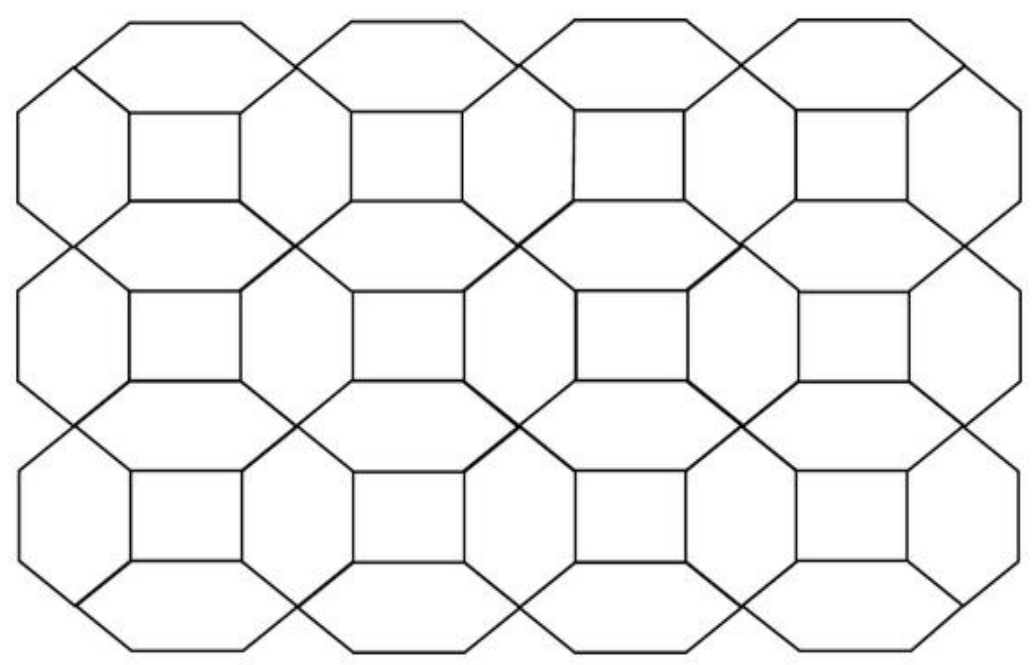

Figure 13: Two dimensional truncated octahedron tessellation [26]

Voronoi tessellation of body centered cubic lattice require $43.25 \%$ fewer nodes than face centered cubic lattice but it still needs $46 \%$ more nodes according to our solution based on satellite foot-print idea. 


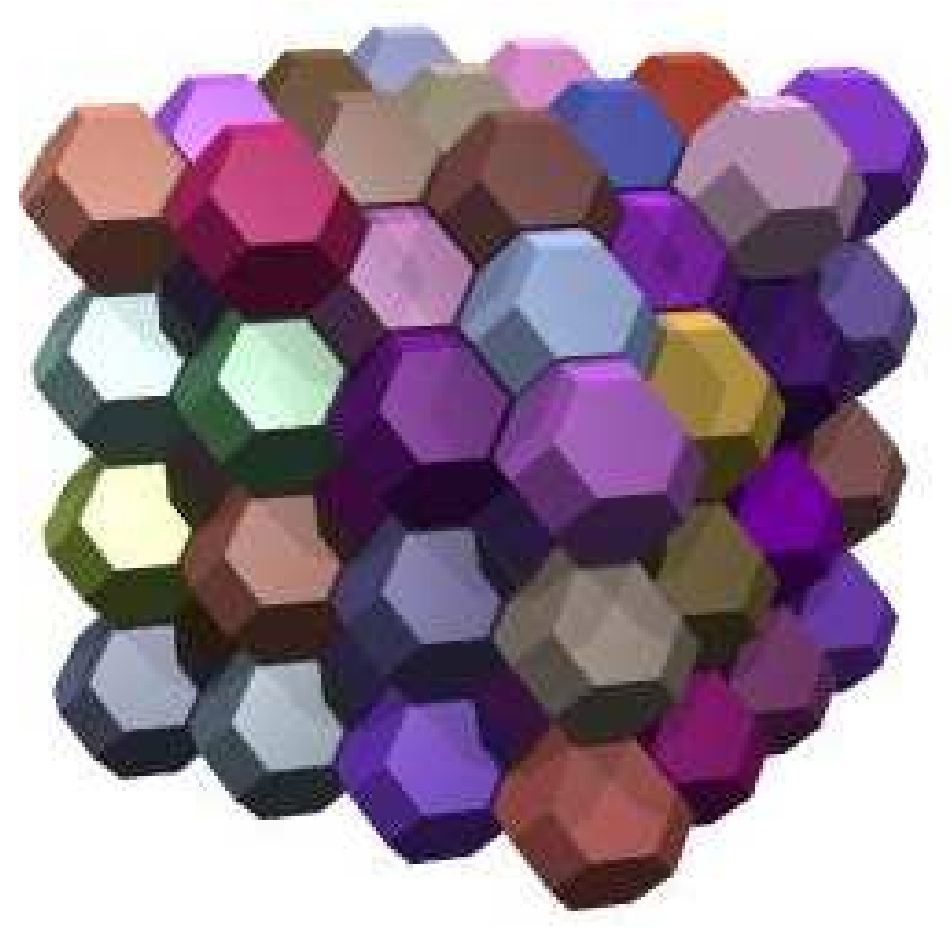

Figure 14: BCC lattice for truncated octahedron in 3 dimensional space [27]

\subsection{Satellite Foot Print}

Satellite foot print means the ground area that its emitter suggests coverage, and decides the satellite diameter needed to collect every emitter's signal. Every emitter or group of emitters have different coverage area. Also every emitter's entire goal is to cover different areas of the ground [28]. But our main goal is to find the shape and if we assume one base station and its coverage. It makes a shape like cone. 


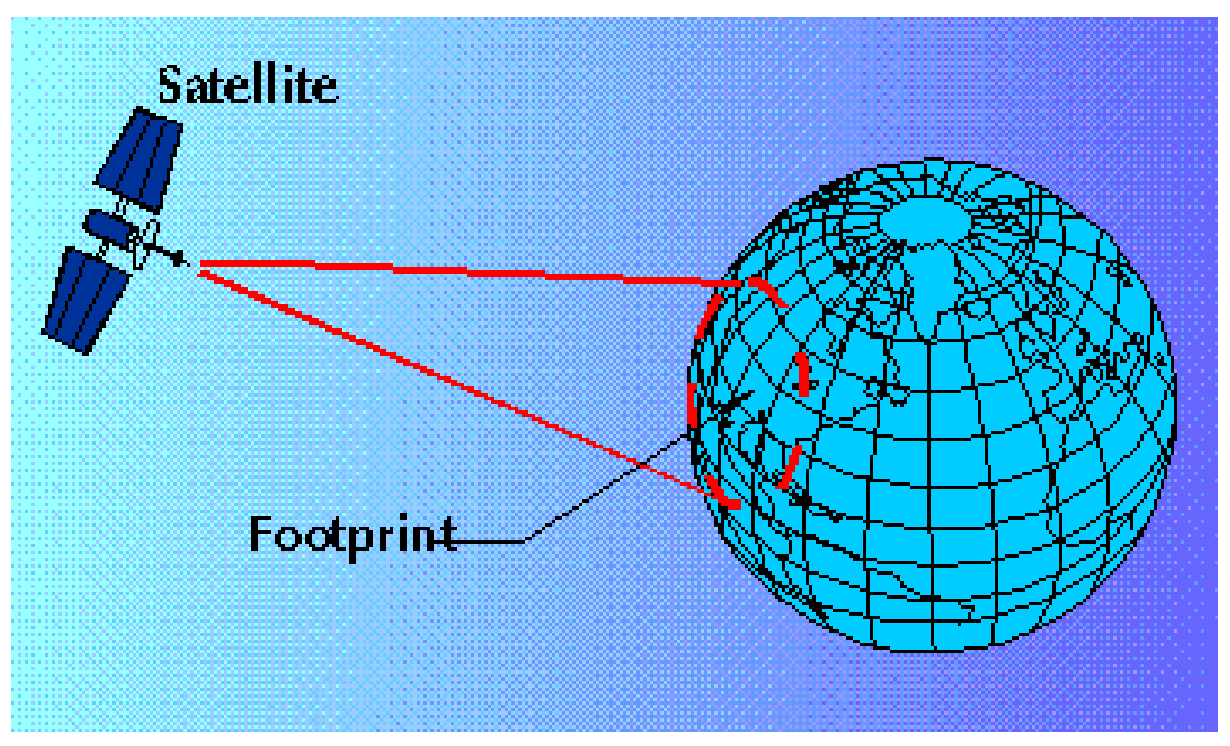

Figure 15: Basic idea of satellite foot-print [29]

Instead of using voronoi tessellation and putting the node in the center of shape, we put the node on the vertex of the cone. This thesis tessellate a three dimensional space with cones. More details about calculation and tessellation will be explained in chapter 3.

\subsection{Assumptions and Goals of Related Works}

Related works made some assumptions for specifying the placement of a node and sensing range.

Assumptions are,

- Every sensor node has the same sensing range $R$. Sensing range is multi directional and every node has a sensing area which is the radius of sphere $R$. Also it has the node at its center [2].

- The constraints for three dimensional area like the length, the width, and the height should be greater than $R$, in order that the boundary effect is not important because of this it can be overlooked [2].

- Every position in the three dimensional area to be filled must be within the sensing area from as a minimum one node [2]. 
- The nodes positions are constant, their position is discretionary. When the nodes are mobile, at the beginning nodes are arbitrarily arranged, and the nodes movement is unlimited. As a result, previous works disregard the physical constraints of placement of the nodes, and previous works suppose that the placement strategy can be changed to deploy a node at some location in the network [2].

Aims are,

- For specific $R$, the number of nodes and their locations are found, in the sense that the number of nodes needed to fill any specific volume is reduced [2].

- For placement strategies that realize the aim above, they calculated the minimum transmission range, in the sense that the all nodes can connect with their adjacent nodes [2].

\subsection{Volumetric Quotient}

Previous works used voronoi cell and they assumed these cells are the same and the border effect are insignificant, in that case the total number of nodes needed for three dimensional coverage area can be found by calculating the ratio of the volume of the three dimensional area to be filled to the volume of voronoi cell. Here we used satellite foot print idea; we put our node to vertex of cone. It is not like to putting the node to the center of the three dimensional shape like voronoi cell but we used the same idea. As a result less number of nodes can be achieved by our cone tessellation approach. There is only one way to achieve less number of nodes. We must have the highest volume for the specific sensing radius $R$. In other words, sensing range $R$ must be greater than the radius of the circumsphere of our cell.

The main goal is achieving the highest volume. The sensing range $R$ must be the same as the radius of circumsphere. Because the $R$ is permanent, the cell has same 
volumes of circumspheres and its constant which is equal to the volume of sphere $4 / 3\left(\pi r^{3}\right)$.

The form of the every cone cell in three dimension is a shape, not a polyhedron. Here the limit is each same cone cell shows that the cone must cover a three dimensional area like polyhedrons. Our approach finds the space filling shape rather than polyhedrons that has the highest ratio. This ratio can be achieved by dividing the shape's volume to volume of its circumsphere. This formula is called volumetric quotient of the shape. Formal definition of volumetric quotient is in this manner:

Definition: "For any polyhedron, if the maximum distance from its center to any vertex is $R$, and the volume of that polyhedron is $V$, then the volumetric quotient of the polyhedron is given as:

$$
\frac{V}{\frac{4}{3} \pi R^{3}}
$$

which is the ratio of the volume of a polyhedron to the volume of its circumsphere." [2]

The volumetric quotient is always between zero and one. In other words, any constant sensing range $\mathrm{R}$, the amount of nodes needed to fill a three dimensional area is inversely proportional to the volumetric quotient of the shape.

Our approach found an alternative solution to find the shape which has the highest volumetric quotient. We achieved one, which represents the highest volumetric quotient with using three dimensional cone. It is not a polyhedron but combining cones can fill a space and there is a figure that you can see it in two dimensions with different number of nodes and their neighbours. 


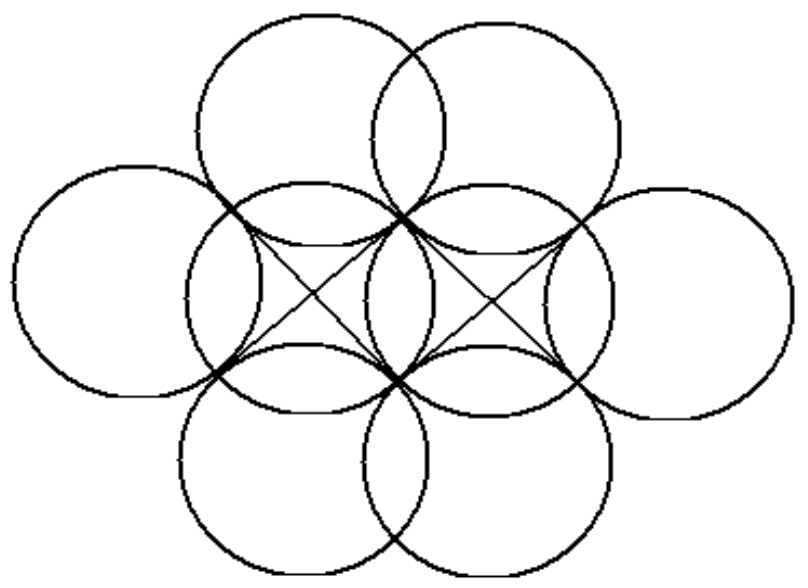

Figure 16: Combination of 4 nodes

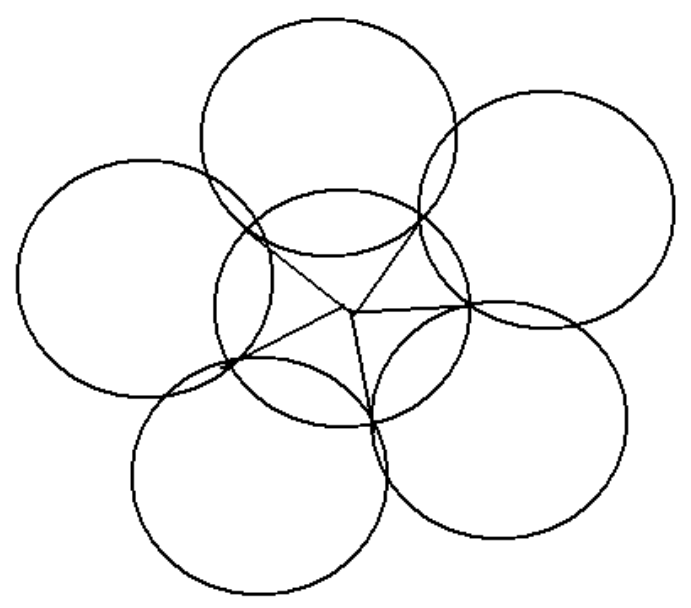

Figure 17: Combination of 5 nodes 


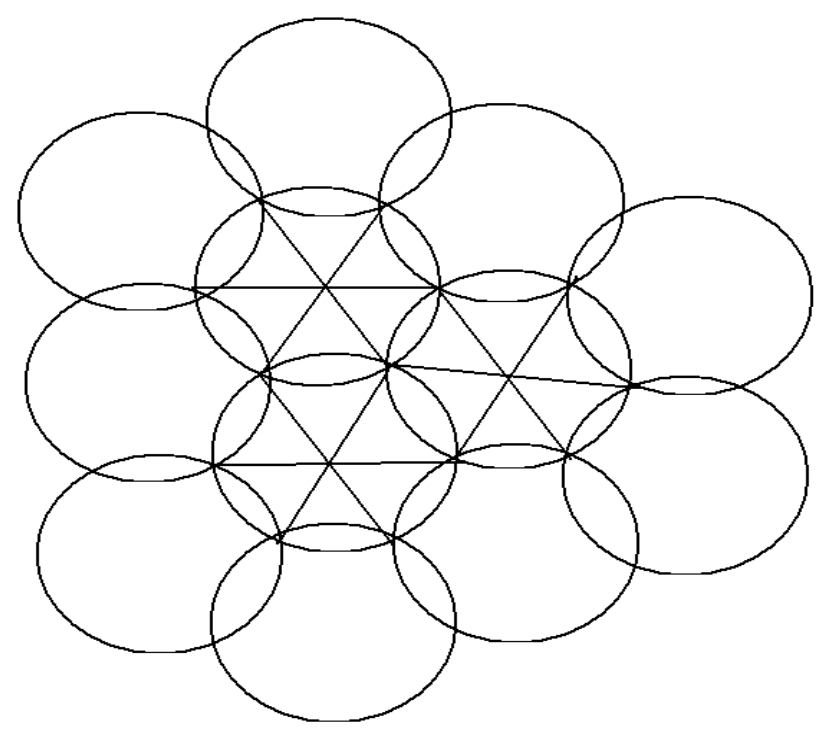

Figure 18: Combination of 6 nodes

Finding optimal shapes take centuries. Kepler's problem solved after five hundred years and Kelvin's conjecture is still an open subject. This thesis compared five polyhedrons and it showed that cone has highest volumetric quotient than other models. As a result cone needs less number of nodes than other models for three dimensional networks. Also connectivity issue depends on finding minimum transmission range to keep up connectivity between adjacent nodes.

\subsubsection{Volumetric Quotients for Polyhedrons}

There are volumetric quotients for previous researches. In Chapter 3, we will compare them with our cone approach.

Volumetric Quotient for Cube:

- $\quad$ Every side of cube has a length which is $a$.

- Radius of circumsphere of cube is $\sqrt{3} a / 2$.

- $\quad$ volumetric quotient $=\frac{a^{3}}{\frac{4}{3} \pi(\sqrt{3} / 2 a)^{3}}=0.36755$ 


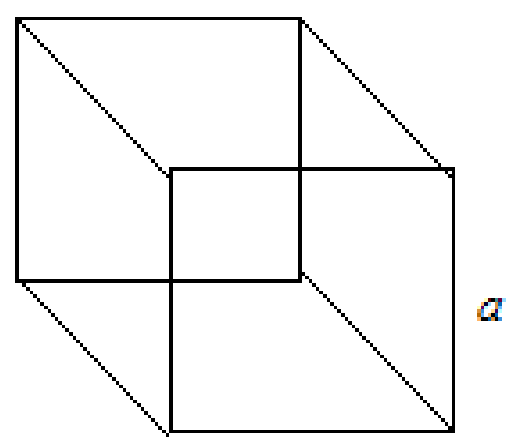

Figure 19: Cube

Volumetric Quotient for Hexagonal Prism:

- Every side of hexagon has a length which is $a$.

- Hexagonal prism's height is $h$.

- Radius of circumsphere is $\sqrt{a^{2}+h^{2} / 4}$.

- volumetric quotient $=\frac{\frac{3 \sqrt{3}}{2} a^{2} a \sqrt{2}}{\frac{4}{3} \pi\left(\sqrt{a^{2}+\frac{a^{2}}{2}}\right)^{3}}=\frac{6}{4 \pi}=0.477$

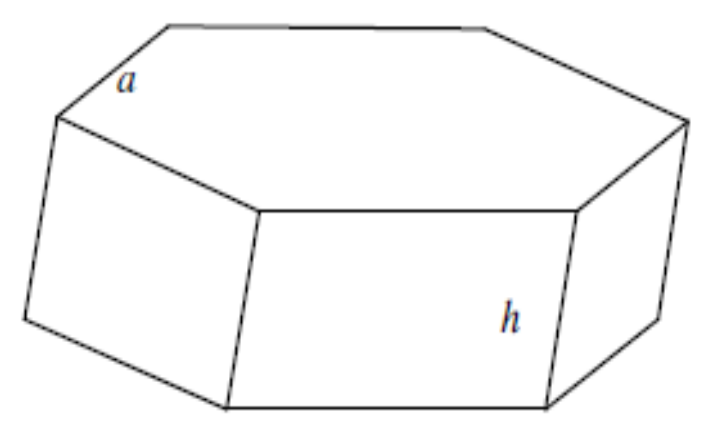

Figure 20: Hexagonal Prism [2]

Volumetric Quotient for Rhombic Dodecahedron:

- $\quad$ Every edge of rhombic dodecahedron has a length which is $\sqrt{3} a / 2$.

- Rhombic dodecahedron's volume is $2 a^{3}$.

- Circumradius of a rhombic dodecahedron is $a$. 
- $\quad$ Volumetric quotient $=\frac{2 a^{3}}{\frac{4}{3} \pi a^{3}}=\frac{6}{4 \pi}=0.477$

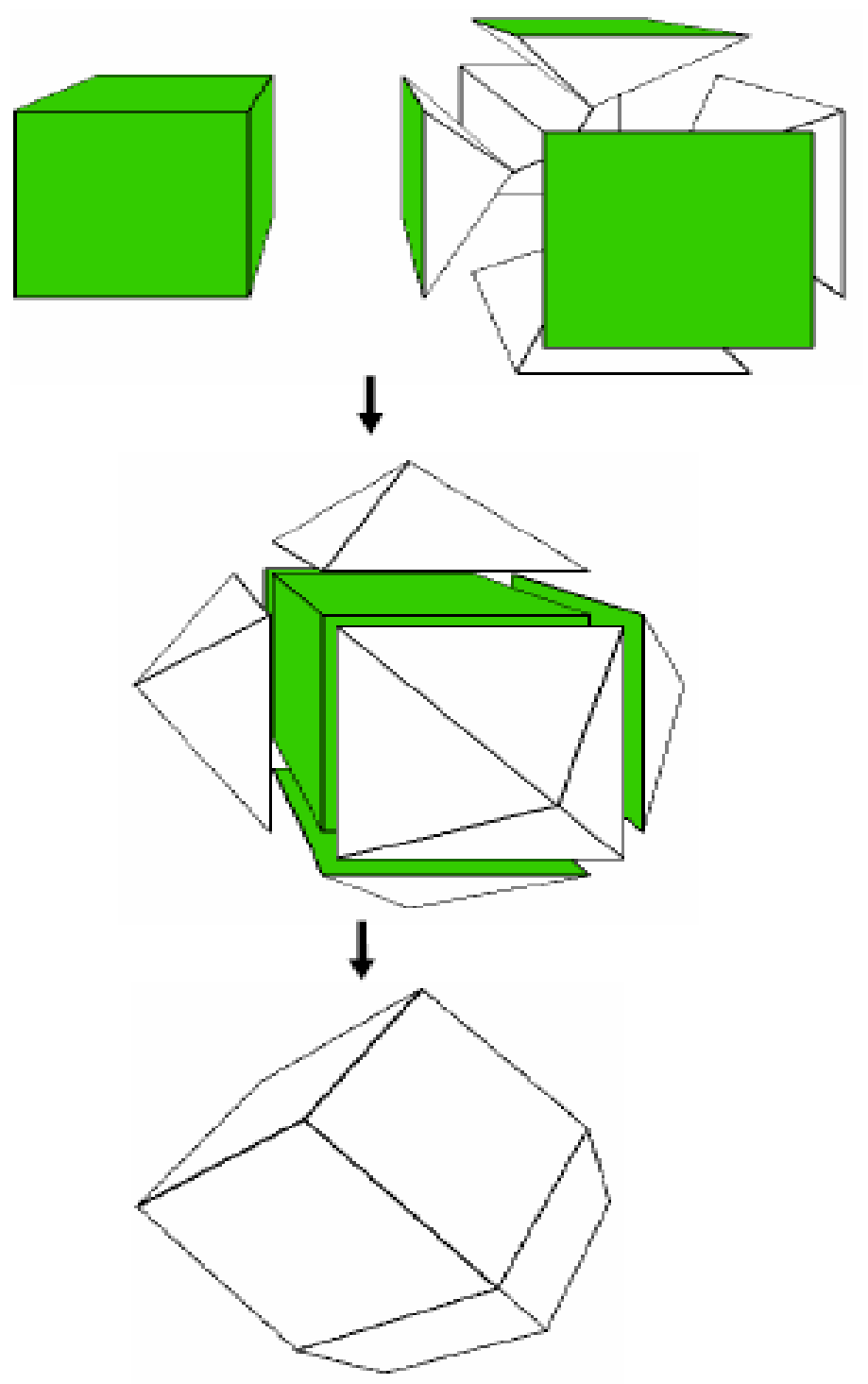

Figure 21: Rhombic dodecahedron [2]

Volumetric Quotient for Truncated Octahedron:

- Every edge of truncated octahedron has a length which is $a$.

- Truncated octahedron's volume is $8 \sqrt{2} a^{3}$.

- Circumradius of truncated octahedron is $\sqrt{10} / 2$. 
- $\quad$ volumetric quotient $=\frac{8 \sqrt{2} a^{3}}{\frac{4}{3} \pi\left(\frac{1}{2} \sqrt{10} a\right)^{3}}=\frac{24}{5 \sqrt{5} \pi}=0.68329$
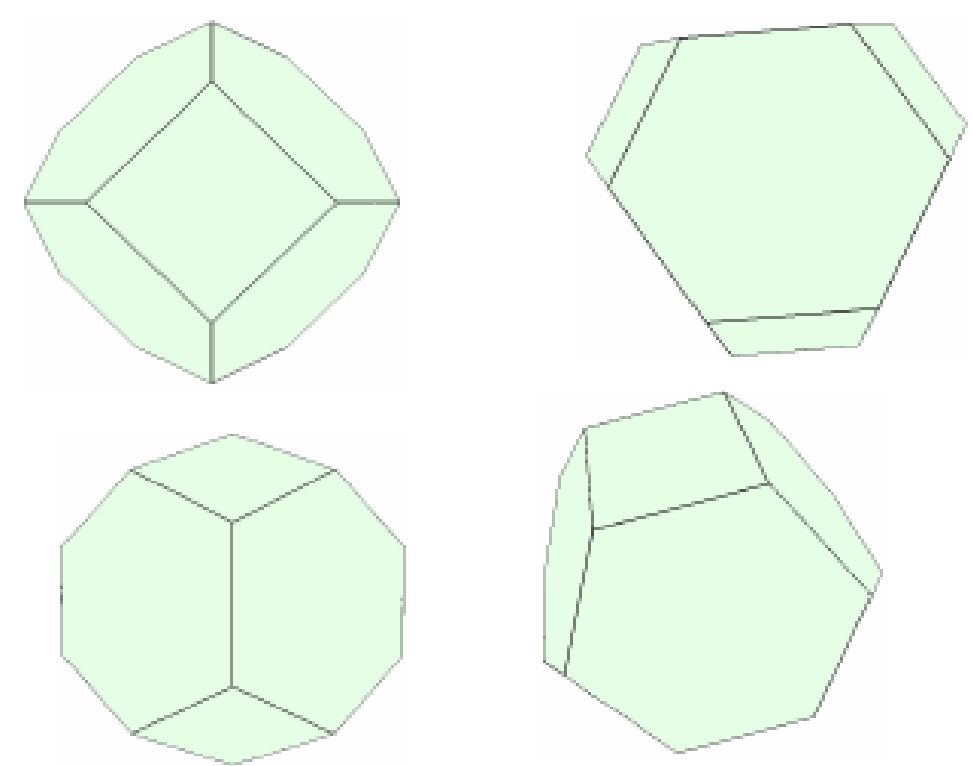

Figure 22: Truncated Octahedron [2]

The results of volumetric quotients are summarized in Table 1.

Table 1: Volumetric quotients for different polyhedrons [2]

\begin{tabular}{|c|c|c|}
\hline Model & $\begin{array}{c}\text { Volumetric } \\
\text { Quotient }\end{array}$ & $\begin{array}{c}\text { Amount of Nodes } \\
\text { Required Compared } \\
\text { to Truncated } \\
\text { Octahedron (\%) }\end{array}$ \\
\hline Cube & 0.36755 & 185.9 \\
\hline Hexagonal Prism & 0.477 & 143.25 \\
\hline $\begin{array}{c}\text { Rhombic } \\
\text { Dodecahedron }\end{array}$ & 0.477 & 143.25 \\
\hline $\begin{array}{c}\text { Truncated } \\
\text { Octahedron }\end{array}$ & 0.68329 & same \\
\hline
\end{tabular}

Volumetric quotient approach of our proposed model will be compared in Chapter 4. 


\subsection{Placement Strategies for Polyhedrons}

In this section, the results for the placement of a node in the coordinate system explained briefly. Previous works put the nodes to the center and coordinates are calculated according to this center node. We should remember that sensing range is $\mathrm{R}$, and coordinate points are shown with $c x, c y$ and $c z$, according to real coordinate system $(x, y, z)$. More details are able to be found in reference [1] and [2]. Placement strategies for previous models explained in subsections below:

\subsubsection{Placement Strategy for Cube}

The distance between every adjacent nodes is $2 R / \sqrt{3}$. Cube tessellation can be obtained by deploying nodes on a three dimensional coordinate system. Three dimensional coordinate system has three axes $(u, v, w)$ and they are parallel to $x, y, z$ axes. This coordinate system have a center which is in terms of $c x, c y, c z$ and unit distance in every axes is $2 R / \sqrt{3}$.

Nodes should be placed at $\left(x+u \frac{2 R}{\sqrt{3}}, y+v \frac{2 R}{\sqrt{3}}, z+w \frac{2 R}{\sqrt{3}}\right)$ and the real distance among any two points in the $u, v, w$ coordinate system is

$d_{12}^{C B}=\frac{2}{\sqrt{3}} R \sqrt{\left(u_{2}-u_{1}\right)^{2}+\left(v_{2}-v_{1}\right)^{2}+\left(w_{2}-w_{1}\right)^{2}}$

\subsubsection{Placement Strategy for Hexagonal Prism}

Researchers assume that all hexagons are placed at plane $x y$ and they are parallel to each other. The distance among adjacent nodes in the direction of $z$ axis is $2 R / \sqrt{3}$. Optimal height for hexagonal prism can be calculated by multiplying the hexagon's radius by $\sqrt{2}$. Hexagonal prism tessellation can be obtained by deploying nodes on a three dimensional coordinate system. The unit distance is $R \sqrt{2}$ for $u$ and $v$ axes and the unit distance is $2 R \sqrt{2}$ for $w$ axis. 
Node should be placed at $\left(x+u R \sqrt{\frac{3}{2}}, y+(u+2 v) \frac{R}{\sqrt{2}}, z+\frac{2 R w}{\sqrt{3}}\right)$ and the real distance among any two points in the $\mathrm{u}, \mathrm{v}, \mathrm{w}$ coordinate system is

$$
d_{12}^{H P}=R \sqrt{2} \sqrt{\left(u_{2}-u_{1}\right)^{2}+\left(u_{2}-u_{1}\right)\left(v_{2}-v_{1}\right)+\left(v_{2}-v_{1}\right)^{2}+\frac{2}{3}\left(w_{2}-w_{1}\right)^{2}}
$$

\subsubsection{Placement Strategy for Rhombic Dodecahedron}

Rhombic dodecahedron tessellation can be obtained by deploying nodes on a three dimensional coordinate system. The unit distance on every axis is $R \sqrt{2}$.

Node should be placed at $\left(x+(2 u+w) \frac{R}{\sqrt{2}}, y+(2 v+w) \frac{R}{\sqrt{2}}, z+w R\right)$ and the real distance among any two points in the $u, v, w$ coordinate system is

$\mathrm{d}_{12}^{\mathrm{RD}}=\mathrm{R} \sqrt{2}$

$\sqrt{\left(u_{2}-u_{1}\right)^{2}+\left(v_{2}-v_{1}\right)^{2}+\left(w_{2}-w_{1}\right)^{2}+\left(u_{2}-u_{1}\right)\left(w_{2}-w_{1}\right)+\left(v_{2}-v_{1}\right)\left(w_{2}-w_{1}\right)}$

\subsubsection{Placement Strategy for Truncated Octahedron}

Truncated octahedron tessellation can be obtained by deploying nodes on a three dimensional coordinate system. The unit distance for $u$ and $v$ axes is $4 \mathrm{R} / \sqrt{5}$ and for $w$ axis is $2 \sqrt{3} R / \sqrt{5}$.

Node should be placed at $\left(x+(2 u+w) \frac{2 R}{\sqrt{5}}, y+(2 v+w) \frac{2 R}{\sqrt{5}}, z+w \frac{2 R}{\sqrt{5}}\right)$ and the real distance among any two points in the $\mathrm{u}, \mathrm{v}, \mathrm{w}$ coordinate system is

$\mathrm{d}_{12}^{\mathrm{TO}}=\frac{4}{\sqrt{5}} \mathrm{R}$

$\sqrt{\left(u_{2}-u_{1}\right)^{2}+\left(v_{2}-v_{1}\right)^{2}+\left(u_{2}-u_{1}\right)\left(w_{2}-w_{1}\right)+\left(v_{2}-v_{1}\right)\left(w_{2}-w_{1}\right)+\frac{3}{4}\left(w_{2}-w_{1}\right)^{2}}$ 
After placement of nodes, calculation of the minimum transmission range is required to keep up connectivity between nodes. For example, if cube is chosen, the distance among two adjacent nodes is $2 R / \sqrt{3}$. Therefore, transmission range value is $1.1547 R$. If hexagonal prism is chosen, the transmission range value $\sqrt{2} R=1.4142 R$ to keep up connectivity with the adjacent node $u$ and $v$ axes, for $w$ axis transmission range value is $2 R / \sqrt{3}=1.1547 R$. If rhombic dodecahedron is chosen, minimum transmission range value is $R \sqrt{2}=1.4142 R$ for both axes. For truncated octahedron, the minimum transmission range for $u$ and $v$ axes is $4 R / \sqrt{5}=1.7889 R$ and for $w$ axis is $2 \sqrt{3} \mathrm{R} / \sqrt{5}=1.5492 \mathrm{R}$. The comparisons are in Table 2 .

Table 2: Minimum transmission range for previous research models [2]

\begin{tabular}{|c|c|c|c|c|}
\hline \multirow{2}{*}{ Model } & \multicolumn{3}{|l|}{$\begin{array}{l}\text { Value of Minimum } \\
\text { Transmission Range }\end{array}$} & $\begin{array}{c}\text { Maximum of the Minimum } \\
\text { Transmission Range }\end{array}$ \\
\cline { 2 - 4 } & $\boldsymbol{u}$ axis & $\boldsymbol{v}$ axis & $\boldsymbol{w}$ axis & \\
\hline Cube & $1.1547 R$ & $1.1547 R$ & $1.1547 R$ & $1.1547 R$ \\
\hline $\begin{array}{c}\text { Hexagonal Prism } \\
\begin{array}{c}\text { Rhombic } \\
\text { Dodecahedron }\end{array}\end{array}$ & $1.4142 R$ & $1.4142 R$ & $1.1547 R$ & $1.4142 R$ \\
\hline $\begin{array}{c}\text { Truncated } \\
\text { Octahedron }\end{array}$ & $1.7879 R$ & $1.7879 R$ & $1.5492 R$ & $1.4142 R$ \\
\hline
\end{tabular}

In chapter 4, our proposed cone model will be compared with previous polyhedron models.

\subsection{Simulation}

Previous works mostly prefer to write their simulation with $\mathrm{C}$ programming language using OpenGL and they implement placement strategies that they found. If they used the placement strategies formulas, they can cover the total space if the voronoi cells have related space filling polyhedrons. Since OpenGL is graphical, researchers 
viewed the shapes from different viewing angels. It is still hard to understand from images (snapshots taken from the animation). Extra nodes can be deployed according to network size to fill the gaps. Most space filler polyhedron should be chosen for networks to cover whole three dimensional space and make the sensing range as big as possible. However, if we mention about a huge network, we can neglect the boundary effect. From below figures, the idea of tessellation and simulation can be understand clearly.

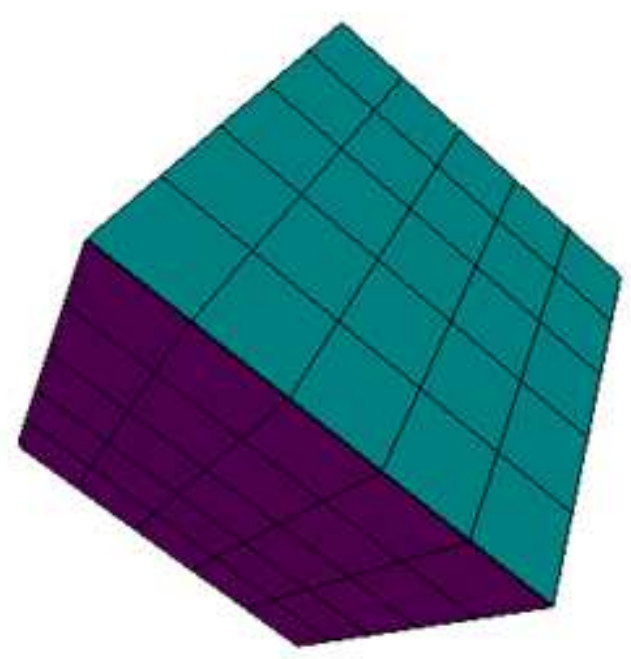

Figure 23: Tessellation for cube

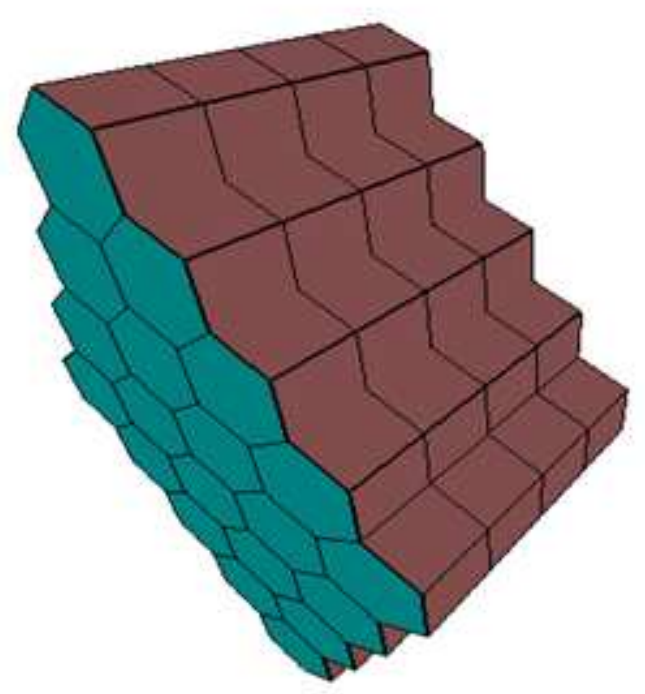

Figure 24: Tessellation for hexagonal prism 


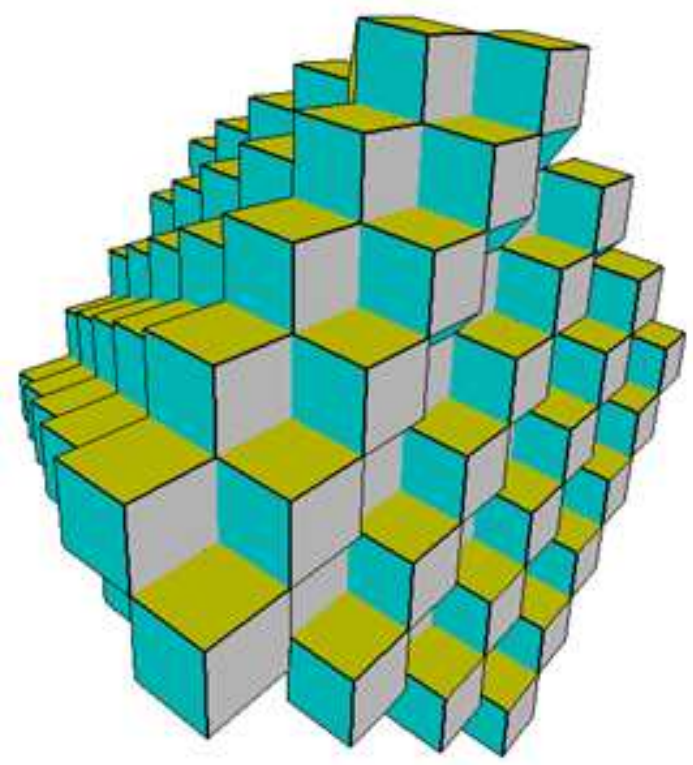

Figure 25: Tessellation for rhombic dodecahedron

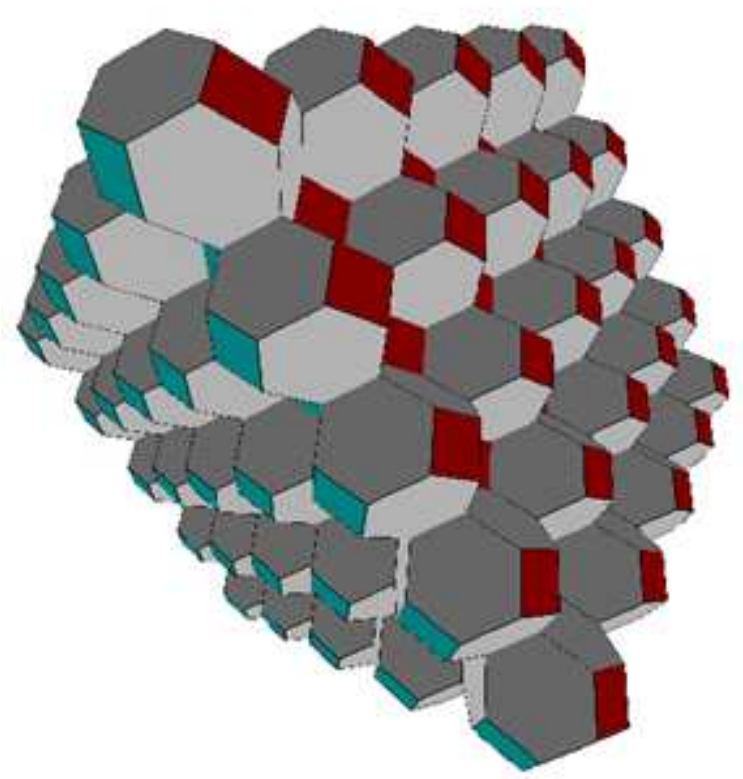

Figure 26: Tessellation for truncated octahedron

If boundaries are given then tessellation can change. Node numbers will increase if the network size increases. In the Figure 27 (a), there are $8 \times 8 \times 8$ nodes and in the Figure 27 (b), there are 20x20x20 nodes for truncated octahedron placement strategy. We should keep in mind that camera positions are changed for screenshot (a) and (b). 

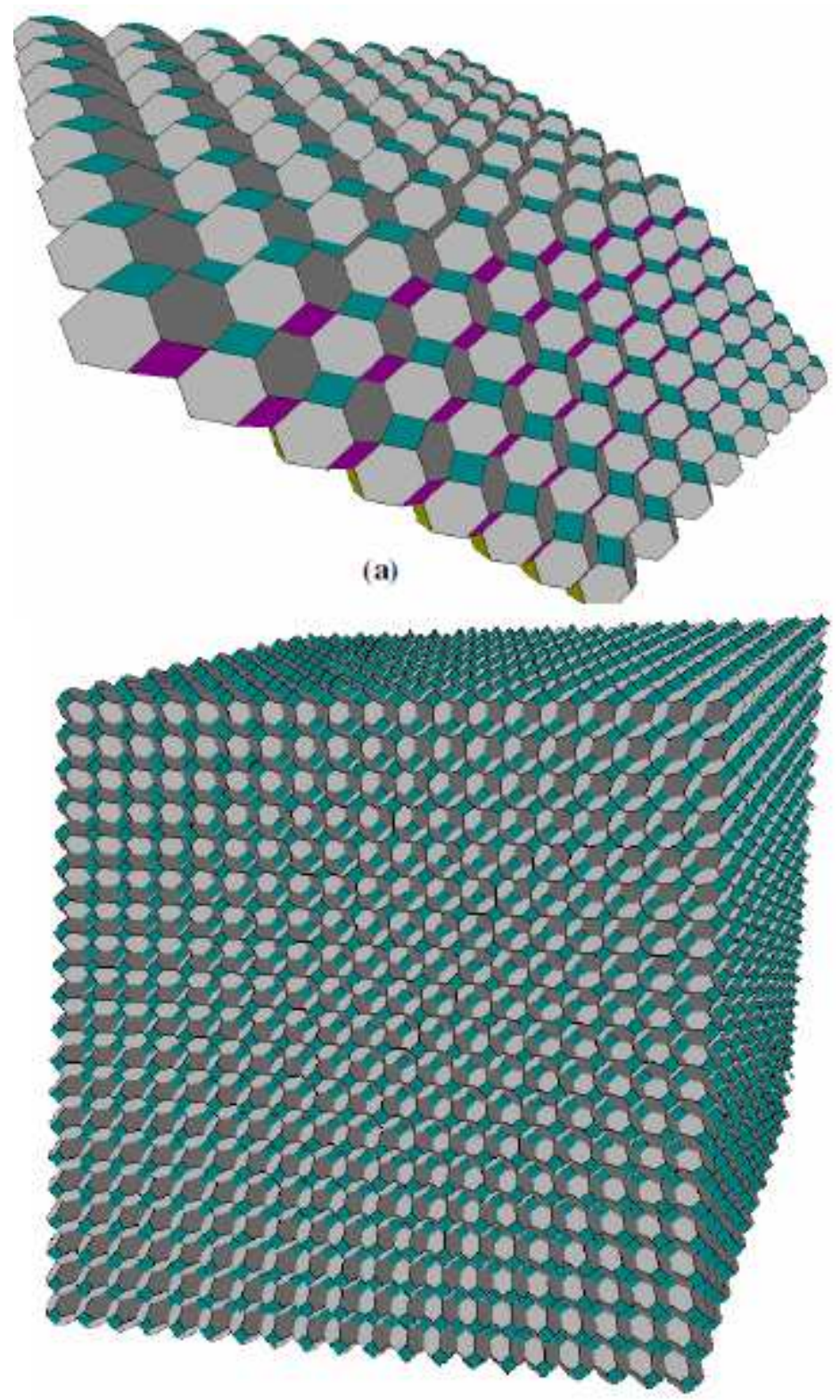

(b)

Figure 27: Tessellation for different network size (a) has 8x8x8 nodes and (b) has $20 \times 20 \times 20$ nodes [2]

\subsection{Related Works about Coverage in History}

For two dimension cellular networks, one hundred percent coverage was guaranteed.

For two dimensional cellular systems, cells are made of identical hexagons. Thus maximum range of emitter is the same as hexagon's radius. For sensor network, sensing coverage is the most important issue. Sensing area should be maximized for lots of applications of sensor networks like targeting, detection and monitoring. All 
points in three dimensional region must be sensed at least one sensor to achieve fully covered network. For two dimensional networks, random network topology can be used. Therefore some algorithms have been developed to get full sensing coverage.

Sensor network's lifetime is another fundamental issue. For increasing lifetime, many energy conservation protocols were developed. So sensing area can be easily managed by setting sensors sleep mode or active mode at a particular time. Also greedy geographic routing is another way to increase sensing coverage for two dimensional wireless sensor networks.

Three dimensional networks is a very new subject, therefore there are few researches about it. In this thesis, cone is used to determine the shape of a cell. Also total number of nodes is $46.35 \%$ fewer than last model that has been found.

Based on our research done so far, we believe that previous models can be implemented in real life. The reason is because, if we want to have the best coverage, we can combine many spheres to form our desired shape as was shown in the some figures. However, the only problem we might face is that, we have to take each and every one of the nodes and place them to get our desired shape so as to obtain geometry. 


\section{Chapter 3}

\section{THE PROPOSED CONE MODEL}

This chapter presents a new cone model which will be explained in detail. Section 3.1 presents general information for cone model. Optimal height for getting better volumetric quotient was shown in Section 3.2. Section 3.3 presents the satellite foot print idea. Section 3.4 mentions the placement for cone model. Section 3.5 shows tessellation in three dimensions. Lastly Section 3.6 explains why cone model is better than other models.

\subsection{General Information about the Proposed Cone Model}

Assume that every node has identical sensing range $R$. Radius of the sphere is $R$, and sensor nodes are placed on vertex. Neighboring nodes should be placed on vertex also. Boundary effects are negligible. Every point in the three dimensional area should be placed in the sensing range $R$ and all points must be covered by at least one node. If $R$ is given, then it is easy to find the total number of nodes. Placement strategy for cone is for finding the minimum number of the transmission range. This range value must be in terms of the sensing range $R$. (Every node must be connected to their adjacent). We used volumetric quotient formula idea to find the three dimensional shape to fill the sphere with less gap.

Recall that volumetric quotient is less than 1 for five specific polyhedron. Our model can achieve the highest volumetric quotient which is 1 , without using any polyhedron. Finding the optimal shape is very hard in three dimensions, it can take 
many years to prove like Kepler's conjecture (it still has $99 \%$ certain of the correctness). So we can accept it as a theorem. We compared our model with four polyhedron shapes and found out that cone has the highest volumetric quotient. So, cone needs less number of nodes than other space filling polyhedrons for coverage in three dimensional networks. Lastly, we developed a placement strategy for cone and then we worked on connectivity issue for finding the minimum transmission range required to achieve connectivity between adjacent nodes in the placement strategy.

\subsection{Optimal Height for Cone Model}

For achieving the highest volumetric quotient, volume formulas should be analyzed very carefully. Radius of cone and height of cone are variables and we had to find the optimal values for both of them. We can see the optimal height, volume of cone and circumsphere of cone and finally we can see the volumetric quotient approach below.

- $\quad$ optimal height for cone is $h=\frac{r}{2}$

- $\quad$ volume of cone is $=\frac{1}{3} \pi r^{2} h$

- $\quad$ circumsphere of cone is equal to $R=h$

- $\quad$ volumetric quotient approach $=\frac{\frac{1}{3} \pi r^{2} R}{\frac{4}{3} \pi R^{3}}=\frac{r^{2}}{4 R^{2}}=1$

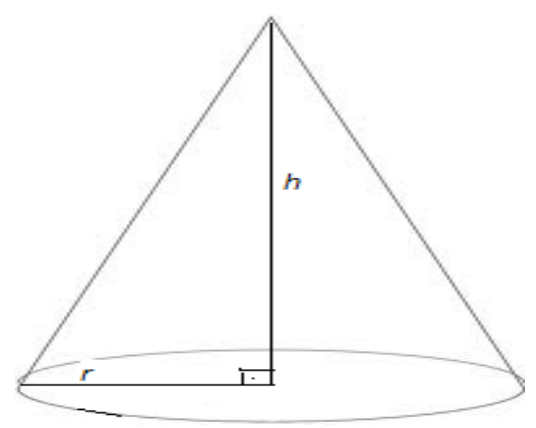

Figure 28: Cone

Here, volumetric quotient can be found as 1, if we divide cone's volume to its sphere's volume with optimal height and optimal radius for our sphere. 


\subsection{Idea of Satellite Foot Print}

Based on satellite foot print idea, we found optimal location for cone as its vertex. When we tile the space and intersect the vertices, we can eliminate one of the nodes and it helped us with volumetric quotient to use less number of nodes.

In the latest research for coverage area in wireless sensor networks, they found that octagon does not tile a plane in two dimensions, blue part is empty and we can easily see it from Figure 29.

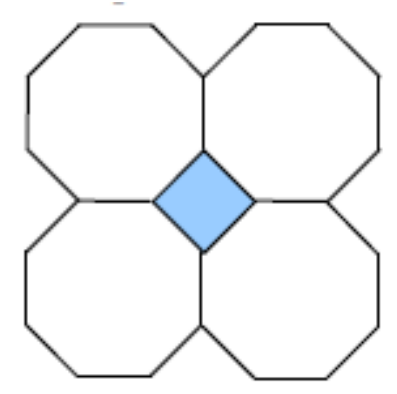

Figure 29: Octagon does not tile a plane [2]

But cone does tile a plane in two dimensions with a specific deployment. Bottom of one cone should stick to bottom of another cone and also vertex of one cone should stick to vertex of another cone. If vertices intersect, we should eliminate one of the nodes.

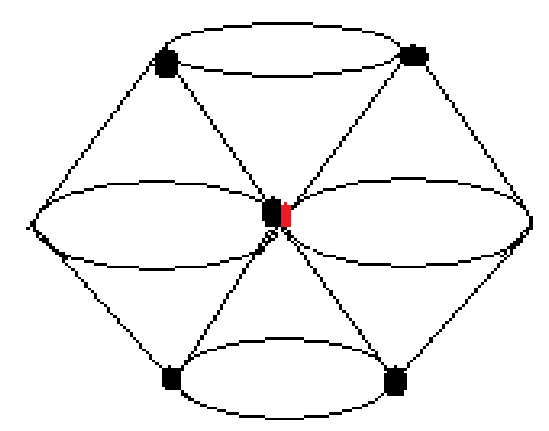

Figure 30: Elimination of the two duplicated nodes in the same coordinates 
If two vertices intersect at the same coordinates, we can eliminate one node at the intersection points thereby reducing the cost of sensors. In addition, the same three dimensional area can be covered if we discard the red node.

\subsection{Placement Strategy for Cone Model}

We placed nodes at vertex, and then we calculated the location of the node according to $x, y$ and $z$ axis.

Assume that the coordinate system has three axes. These are $u, v$ and $w$ axes and they are parallel to real coordinate system which is $x, y$ and $z$ axes. For a node, unit distance in $u$ direction is $2 R, v$ axis is $R$ and $w$ axis is $\sqrt{(2 R)^{2}+R^{2}}$ which is equal to $\sqrt{5} R$.

In addition, node is placed at $(x+u 2 R, y+v R, z+w \sqrt{5} R)$. Optimal height for cone to achieve highest volumetric quotient is $r / 2 . R$ is the sensing range and it is equal to $\mathrm{h}$ for cone $(R=h)$ and the real distance among any two points in the $u, v, w$ coordinate system is

$$
d_{12}^{C N}=R \sqrt{4\left(u_{2}-u_{1}\right)^{2}+\left(v_{2}-v_{1}\right)^{2}+5\left(w_{2}-w_{1}\right)^{2}}
$$

Note that if nodes are placed on the same axis, distance formula is not valid for them because they only have two dimensions in this case. Also we should take the closest neighboring node. Only distance of blue lines can be calculated by cone distance formula in Figure 31. 


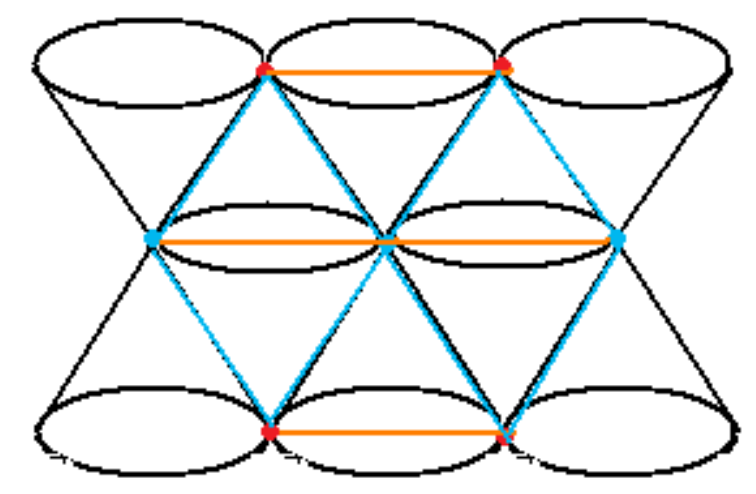

Figure 31: Validity of distance formula

\subsection{Tessellation for Cone Model in Three Dimensions}

There is a tessellation model for better understanding the node placement strategy and it is done by 3Ds Max. It is a .avi file and everyone can see the tessellation with media player. More nodes are required to fill the area if network size increases. However for same network size, cone needs less number of nodes to get full coverage. Figure 32 shows the node placement for cone which is done by 3Ds Max. Also in Appendix A, there are screen shots of tessellation step by step.

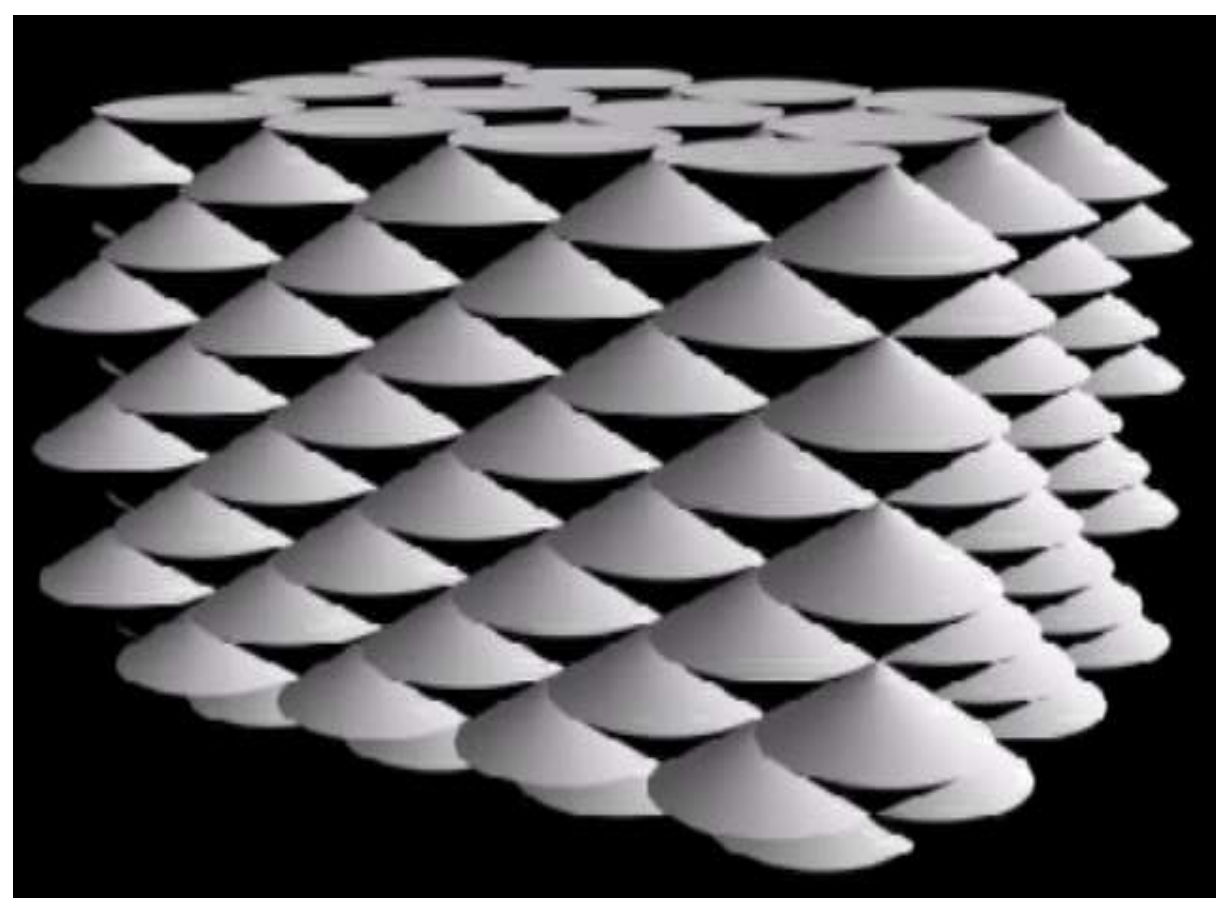

Figure 32: Tessellation for the cone model 


\subsection{Advantage of Cone Model}

Edges of the rhombic dodecahedron and hexagonal prism are hexagons, therefore the sensing range is greater than hexagon's radius. In other words, we can fill a sphere with hexagon but tiling with cone has less gaps. Figure 33 shows the gaps for hexagon and cone in two dimensions. Red places show the gaps.
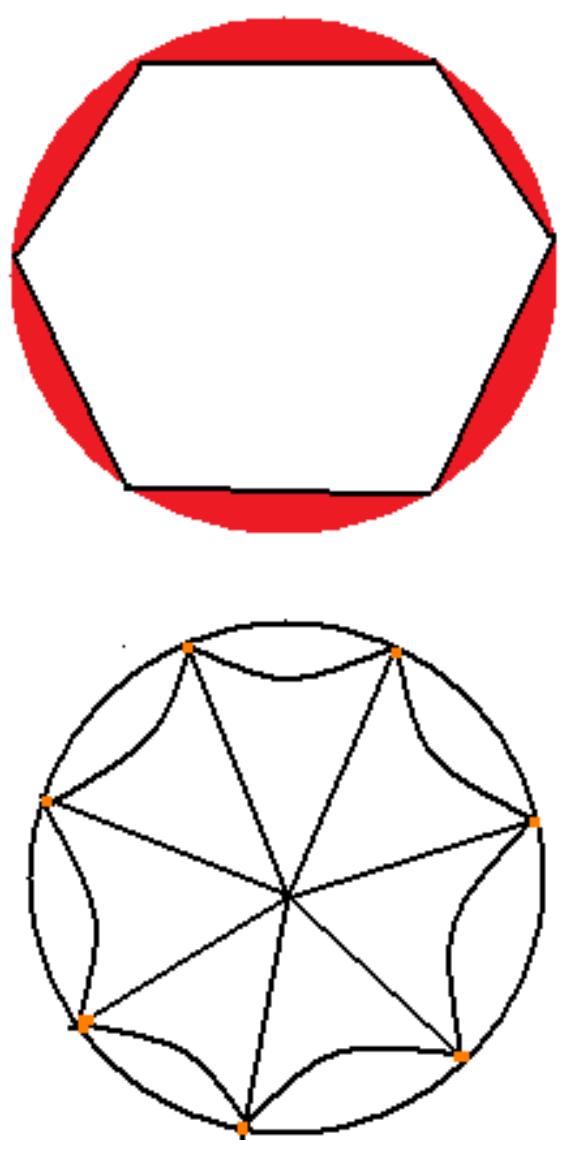

Figure 33: Gaps for Hexagon and Cone 


\section{Chapter 4}

\section{PERFORMANCE ANALYSIS}

This chapter presents the volumetric quotient for different models. The total number of nodes was compared in Section 4.1. Then minimum transmission range for different models was compared in Section 4.2.

\subsection{Comparison of Volumetric Quotients and Number of Nodes}

We showed volumetric quotient for cube model, hexagonal prism model, rhombic dodecahedron model and truncated octahedron model in Chapter 3. Also we calculated volumetric quotient for cone model in Chapter 4. As the value of volumetric quotient increases, the total number of nodes needed for full coverage will be decreased. In that case, we can deploy fewer nodes to the same three dimensional area and that means that we have not to pay more to buy sensor that we do not need to use.

In two dimensions, there is no shape that can fill the plane without gaps; therefore volumetric quotient approach can never be exactly 1 . But we have an advantage in three dimensions. The cells can fill gaps when they are deployed carefully (to have a full filled space). Figure 30 can give an idea about our node deployment strategy. If we have a constant sensing range, cone needs less cells to fill a specific three dimensional space. 
If we consider all models, cone gives the best volumetric quotient value according to our approach. As we mentioned before volumetric quotient is a characteristic issue for determining number of nodes. If we select cube model for deployment, it needs $1 / 0.36755=2.7207$ times that of cone model. For hexagonal prism model, volumetric quotient value is $1 / 0.477=2.0964$ and rhombic dodecahedron model, volumetric quotient value is the same value with hexagonal prism model. In the case of truncated octahedron model, volumetric quotient value is $1 / 0.68329=1.4635$. Table 3 shows the comparisons according to cone model. The achieved saving ratios are shown in the last column.

Table 3. Volumetric Quotients of Models and Number of Nodes Compared to Cone

\begin{tabular}{|c|c|c|c|}
\hline Model & $\begin{array}{c}\text { Volumetric } \\
\text { Quotient }\end{array}$ & $\begin{array}{c}\text { Total Number of } \\
\text { Nodes Required } \\
\text { Compared to Cone }\end{array}$ & $\begin{array}{c}\text { Saving Ratio } \\
\text { Compared to } \\
\text { Cone }\end{array}$ \\
\hline Cube & 0.36755 & 272.07 & $172 \%$ \\
\hline Hexagonal Prism & 0.477 & 209.64 & $110 \%$ \\
\hline $\begin{array}{c}\text { Rhombic } \\
\text { Dodecahedron }\end{array}$ & 0.477 & 209.64 & $110 \%$ \\
\hline $\begin{array}{c}\text { Truncated } \\
\text { Octahedron }\end{array}$ & 0.68329 & 146.35 & $46 \%$ \\
\hline Cone & 1 & 100 & $0 \%$ \\
\hline
\end{tabular}

\subsection{Comparison of Minimum Transmission Ranges for Different}

\section{Models}

For calculating the minimum transmission range value, the angles between real coordinate system and $u, v$ and $w$ axes are the most important issue. If we select cube model, $u, v$ and $w$ axes are parallel to real coordinate system which is $x, y$ and $z$ axes. For hexagonal prism model, there is $30^{\circ}$ difference between $u$ and $x$ axis and there is $60^{\circ}$ difference between $u$ and $y$ axis. Also $w$ axis has $90^{\circ}$ to the $u v$ plane. In the case of rhombic dodecahedron model, $u$ and $v$ axes are parallel to the $x$ and $y$ axes. The 
difference between $u$ and $w$ axes is $60^{\circ}$. Also the difference between $v$ and $w$ is $60^{\circ}$. In addition, the difference between $w$ and $z$ axes is $45^{\circ}$. For truncated octahedron model, again $u$ and $v$ axes are parallel to $x$ and $y$ axes. Also $w$ axis has $54.73^{0}$ with the $z$ axis. In the case of our cone model, $u, v$ and $w$ axes are parallel to real coordinate system $x, y$ and $z$ axes. The difference between $u, v$ and $w$ axes is $90^{\circ}$.

For comparison remind that, minimum transmission range is important to achieve connectivity between adjacent nodes and it depends on the decision of the model. $2 R \sqrt{3}$ is the distance between two adjacent nodes for cube, then the transmission range value is equal to $1.1547 R$. For hexagonal prism, the transmission range is $\sqrt{2} \mathrm{R}=1.4142 R$ along the $u$ and $v$ axes, and $2 R / \sqrt{3}=1.1547 R$ along the $w$ axis. The minimum transmission range value is $\sqrt{2} R=1.4142 R$ for both axes for rhombic dodecahedron model. If we choose the truncated octahedron model, the transmission range value is $4 R \sqrt{5}=1.7889 \mathrm{R}$ along $u$ and $v$ axes, and for $w$ axis this value is $R 2 \sqrt{3} / \sqrt{5}=1.5492 R$

For our proposed cone model, the transmission range value is $2 R$ for $u$ axis, $R$ for $v$ axis and $R \sqrt{5}=2.2360 R$ for $\mathrm{w}$ axis. Table 4 shows the minimum transmission range for these different models. It is clear from the table that the proposed cone model manifests its superiority in terms of transmission range as well. 
Table 4: Comparison of Minimum Transmission Ranges for Different Models

\begin{tabular}{|c|l|l|l|c|}
\hline \multirow{2}{*}{ Model } & \multicolumn{2}{|l|}{$\begin{array}{l}\text { Minimum Transmission } \\
\text { Range }\end{array}$} & $\begin{array}{c}\text { Maximum of the Minimum } \\
\text { Transmission Range }\end{array}$ \\
\cline { 2 - 4 } & u axis & v axis & w axis & \\
\hline Cube & $1.1547 R$ & $1.1547 R$ & $1.1547 R$ & $1.1547 R$ \\
\hline $\begin{array}{c}\text { Hexagonal Prism } \\
\text { Rhombic } \\
\text { Dodecahedron }\end{array}$ & $1.4142 R$ & $1.4142 R$ & $1.1547 R$ & $1.4142 R$ \\
\hline $\begin{array}{c}\text { Truncated } \\
\text { Octahedron }\end{array}$ & $1.7879 R$ & $1.7879 R$ & $1.5492 R$ & $1.4142 R$ \\
\hline Cone & $2 R$ & $R$ & $2.2360 R$ & $1.7889 R$ \\
\hline
\end{tabular}

As we can understand from Table 4, all of the values for the minimum transmission ranges are compared. Then largest value is defined as the maximum of the minimum transmission range. Maximizing the transmission range is necessary. We can think of the idea in this manner: It is obvious that sensing range is always greater than the transmission range. For example if we want to transmit data, we want transmission range value very close to sensing range value. That means, if we maximize the transmission range value it can be very close to sensing range. And we can transmit our data to long distances.

For $u$ and $w$ axes, we achieved better values than other models. In the direction of $w$ axes we get the value which is 2.2360 times of radius of cone and it's a very good result. Since the closest minimum transmission range value is 1.7879 , which belongs to truncated octahedron model. But we achieved a result, which is 1.2509 times more than truncated octahedron model. 


\section{Chapter 5}

\section{CONCLUSION}

This thesis searched three dimensional networks and started to work on connectivity and coverage issues. There is not much resources to research because three dimensional networks is a very new research area. We were inspired from volumetric quotient approaches from previous models and we combined it with satellite foot print idea. Also we explained the previous models. Their shapes, volumetric quotients and minimum transmission ranges were shown. In addition, we compared the results with our proposed cone model and we found that our model is the best model for maximizing the coverage and finding the maximum of minimum transmission range to achieve full connectivity.

The proposed cone model placed nodes in three dimensional space, unlike today's networks (where they assume nodes are placed on two dimensional plane). Deployment of the nodes is not very easy in three dimensions. For two dimensional networks, hexagonal tiling is the best strategy for placing the base stations, so the covered area is maximized with fixed radius base stations. Here, the problem for three dimensional space is filling the empty spaces between nodes. Related works on this topic just have the polyhedron shapes to achieve space-filling property in three dimensions. 
This thesis worked on a shape which is not a polyhedron and we showed that any shape different from polyhedron can tessellate a three dimensional area. We used the idea of volumetric quotient that is the ratio of the volume of a polyhedron's volume to its circumsphere's volume, to compare different models. The proposed cone model results in the highest volumetric quotient which is 1 . This shows that with cones, we can cover the sphere better than polyhedrons. Volumetric quotient is characteristic issue for coverage area. Therefore, the total number of nodes needed for coverage is changed based on volumetric quotient. For example, if we apply truncated octahedron placement strategy, it needs $46 \%$ more nodes than the cone placement strategy to cover same network size. From other related models like cube, hexagonal prism, rhombic dodecahedron, the achieved saving in terms of the total number of nodes are $172 \%, 110 \%$ and $110 \%$ respectively.

We investigated the connectivity issues after discovering the efficient placement strategy. This thesis found the best placement strategy with cone model which needs the transmission range value to be 2.2360 times the sensing range with the aim of getting full connectivity. For cube model, hexagonal prism model, rhombic dodecahedron model and truncated octahedron model transmission range values are $1.1547,1.4142,1.4142$ and 1.7889 respectively.

As a future work, simulation model can be done. Also optimization theory can be implemented for determining the number of cones to divide a sphere in efficient way. Applying the k- connectivity properties for cone model can be another research area. We believe that our proposed model can be a good reference for future works in three dimensional networks. 


\section{REFERENCES}

[1] Alam S.M.N, Haas Z. Coverage and Connectivity in three-dimensional underwater sensor networks.Wireless communication and mobile computing. 2008, www.interscience.wiley.com.

[2] Alam S.M.N, Haas Z. Coverage and Connectivity in three-dimensional networks. In Proceedings of ACM MobiCom, 2006.

[3] Akyildiz, I.F., Pompili, D., Melodia, T., Underwater Acoustic Sensor Networks: Research Challenges, Ad Hoc Networks Journal, (Elsevier), March 2005.

[4] John Heidemann, Wei Ye, Jack Wills, Affan Syed, and Yuan Li. Research Challenges and Applications for Underwater Sensor Networking, IEEE Wireless Communications and Networking Conference, p. to appear. Las Vegas, Nevada, USA, IEEE. April, 2006.

[5] Jiejun Kong, Jun-hong Cui, Dapeng Wu, Mario Gerla, Building Underwater Adhoc Networks and Sensor Networks for Large Scale Real-time Aquatic Applications, IEEE Military Communications Conference (MILCOM'05), October 17-20, 2005. Atlantic City, New Jersey, USA.

[6] I. Vasilescu, K. Kotay, D. Rus, M. Dunbabin and P. Corke,Data Collection, Storage, and Retrieval with an Underwater Sensor Network, SenSys'05, November 2-4, 2005, San Diego, California, USA.

[7] Rappaport, T. S., Wireless Communications: Principles and Practice, Prentice Hall, 2002. 
[8] T. Couqueur, V. Phipatanasuphorn, P. Ramanathan, and K. K. Saluja. Sensor deployment strategy for target detection. In Proceeding of The First ACM International Workshop on Wireless Sensor Networks and Applications, pages 169177, Sep 2002.

[9] K. Chakrabarty, S. S. Iyengar, H. Qi, and E. Cho. Grid coverage for surveillance and target location in distributed sensor networks. IEEE Transactions on Computers, 51(12):1448-1453, December 2002.

[10] S. Meguerdichian, F. Koushanfar, M. Potkonjak, and M. B. Srivastava. Coverage problems in wireless ad-hoc sensor networks. INFOCOM'01, pages 13801387, 2001.

[11] H. Zhang and J. C. Hou, Maintaining sensing coverage and connectivity in large sensor networks, Wireless Ad Hoc and Sensor Networks: An International Journal, Vol. 1, No. 1-2, pp. 89--123, January 2005.

[12] D. Tian and N. D. Georganas, A coverage-preserved node scheduling scheme for large wireless sensor networks. In Proceedings of First International Workshop on Wireless Sensor Networks and Applications (WSNA'02), pages 169-177, Atlanta, USA, Sep 2002.

[13] X. Wang, G. Xing, Y. Zhang, C. Lu, R. Pless, and C. D.Gill. Integrated coverage and connectivity configuration in wireless sensor networks. Sensys, 2003.

[14] T. Yan, T. He, and J. A. Stankovic, Differentiated surveillance for sensor networks., SenSys '03: Proceedings of the 1st international conference on Embedded networked sensor systems, 2003. 
[15] F. Ye, G. Zhong, S. Lu, and L. Zhang, Peas: A robust energy conserving protocol for long-lived sensor networks, 23rd International Conference on Distributed Computing Systems (ICDCS'03), pages 169-177, May 2003.

[16] G. Xing, C. Lu, R. Pless, and Q. Huang, On Greedy Geographic Routing Algorithms in Sensing-Covered Networks, In Proc. of MobiHoc'04, Tokyo, Japan, May 2004.

[17] J. Carle, J.F. Myoupo, and D. Semé. A Basis for 3-D Cellular Networks. In Proc. of the 15th International Conference on Information Networking, 2001.

[18] Catherine Decayeux and David Semé: A New Model for 3-D Cellular Mobile Networks, ISPDC/HeteroPar 2004.

[19] mathworld.wolfram.com/KeplerConjecture.html

[20] http://en.wikipedia.org/wiki/Kepler_conjecture

[21] pitt.edu/ thales/kepler_98

[22] http://www.math.psu.edu/qdu/Res/Pic/gallery3.html

[23] http://mathworld.wolfram.com/Honeycomb.html

[24] http://en.wikipedia.org/wiki/File:Bicolor_cubic_honeycomb.png

[25] http://www.qwiki.com/q/\#!/Rhombo-hexagonal_dodecahedron

[26] http://www.hindawi.com/journals/ijdsn/2010/547368/fig4/ 
[27] http://www.all-science-fair-projects.com/science_fair_projects_encyclopedia/ Image:Truncated_octahedra.jpg

[28] http://en.wikipedia.org/wiki/Footprint_(satellite)

[29] http://dictionary.babylon.com/footprint/ 
APPENDIX 


\section{Appendix A: Snapshots of 3Ds Max for Cone Model}

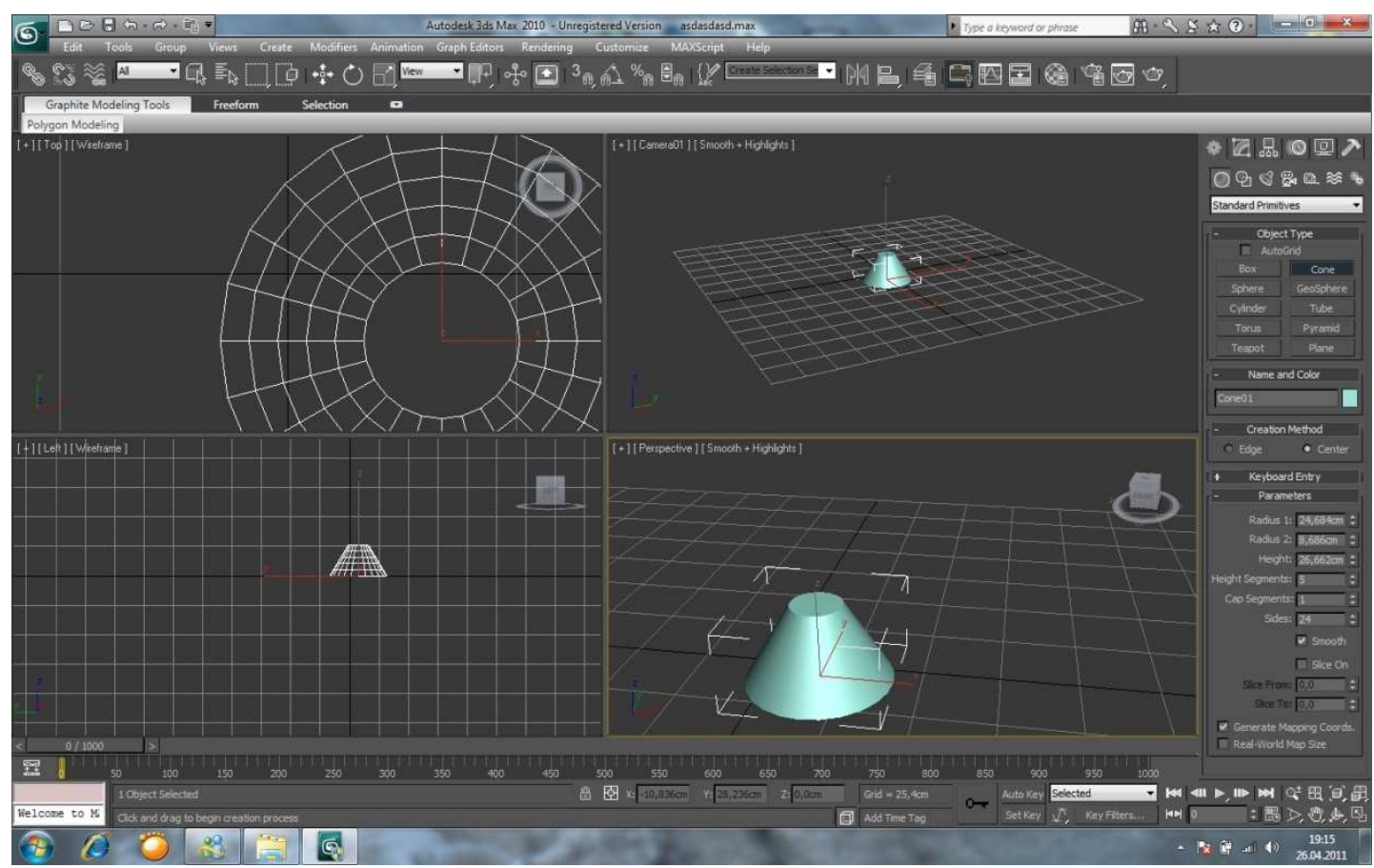

Figure A.1: Constructing a cone in three dimensional space

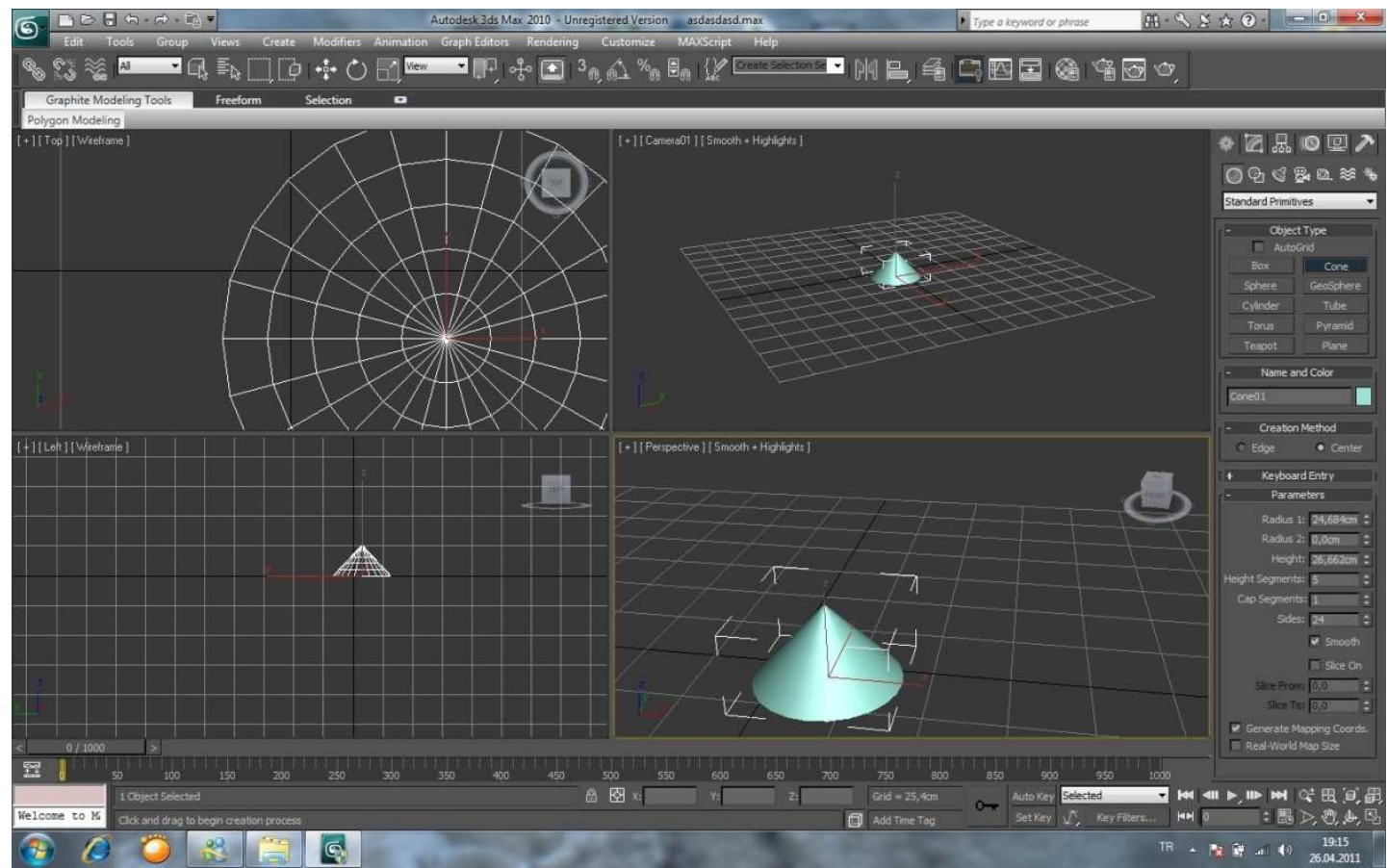

Figure A.2: Placing the cone in three dimensional coordinate system 


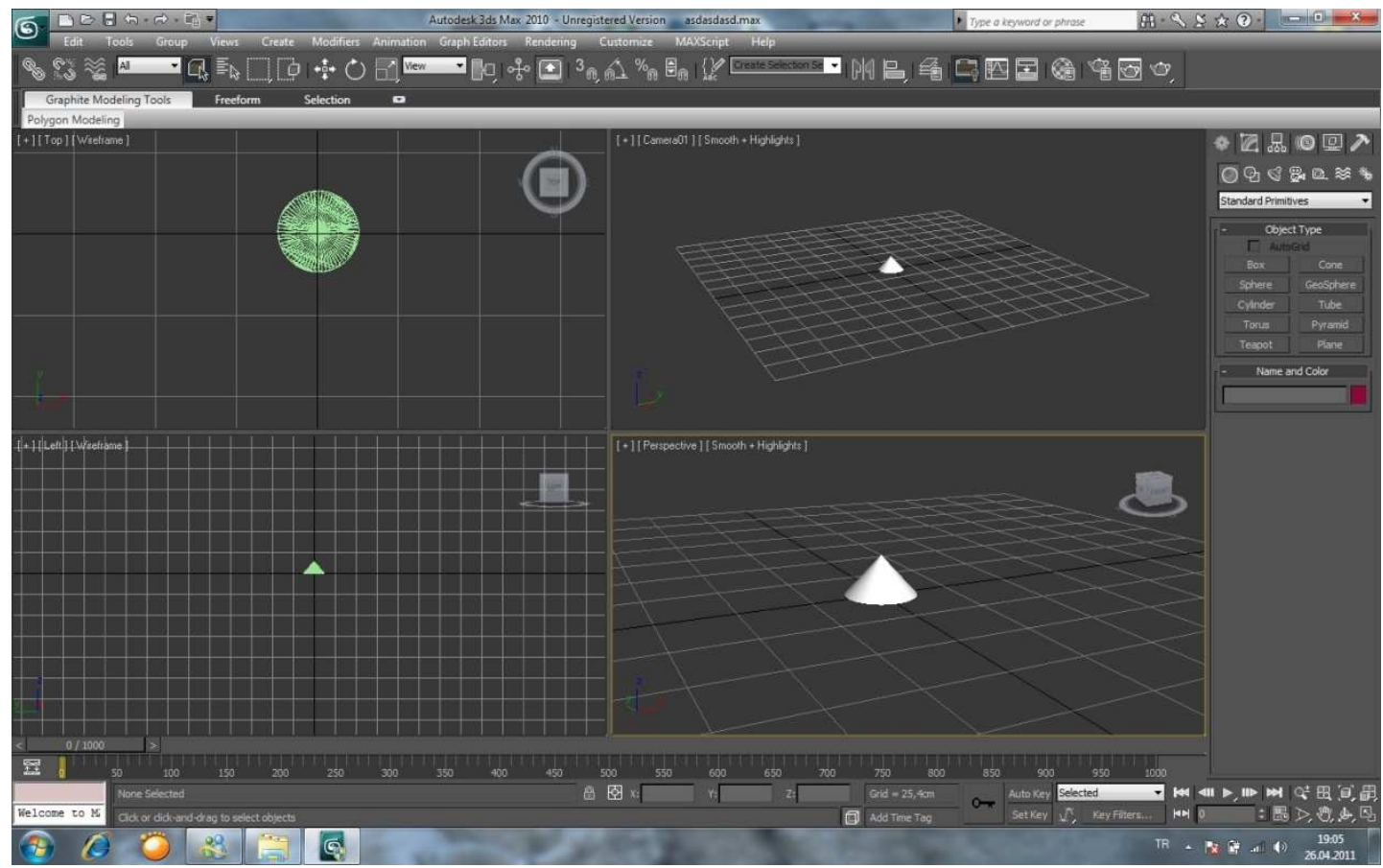

Figure A.3: Specifying the height and the radius of cone

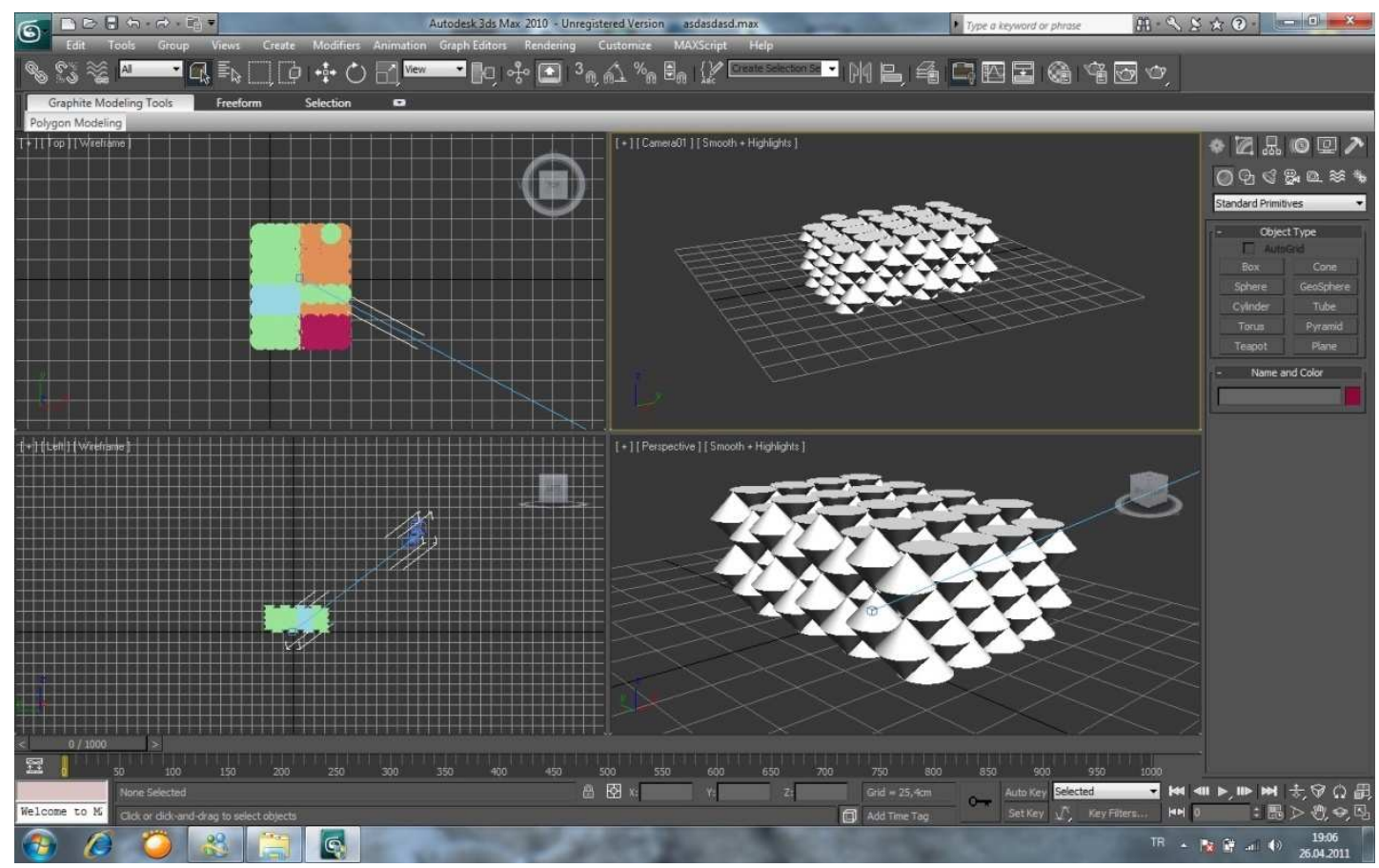

Figure A.4: Determining the place of camera 


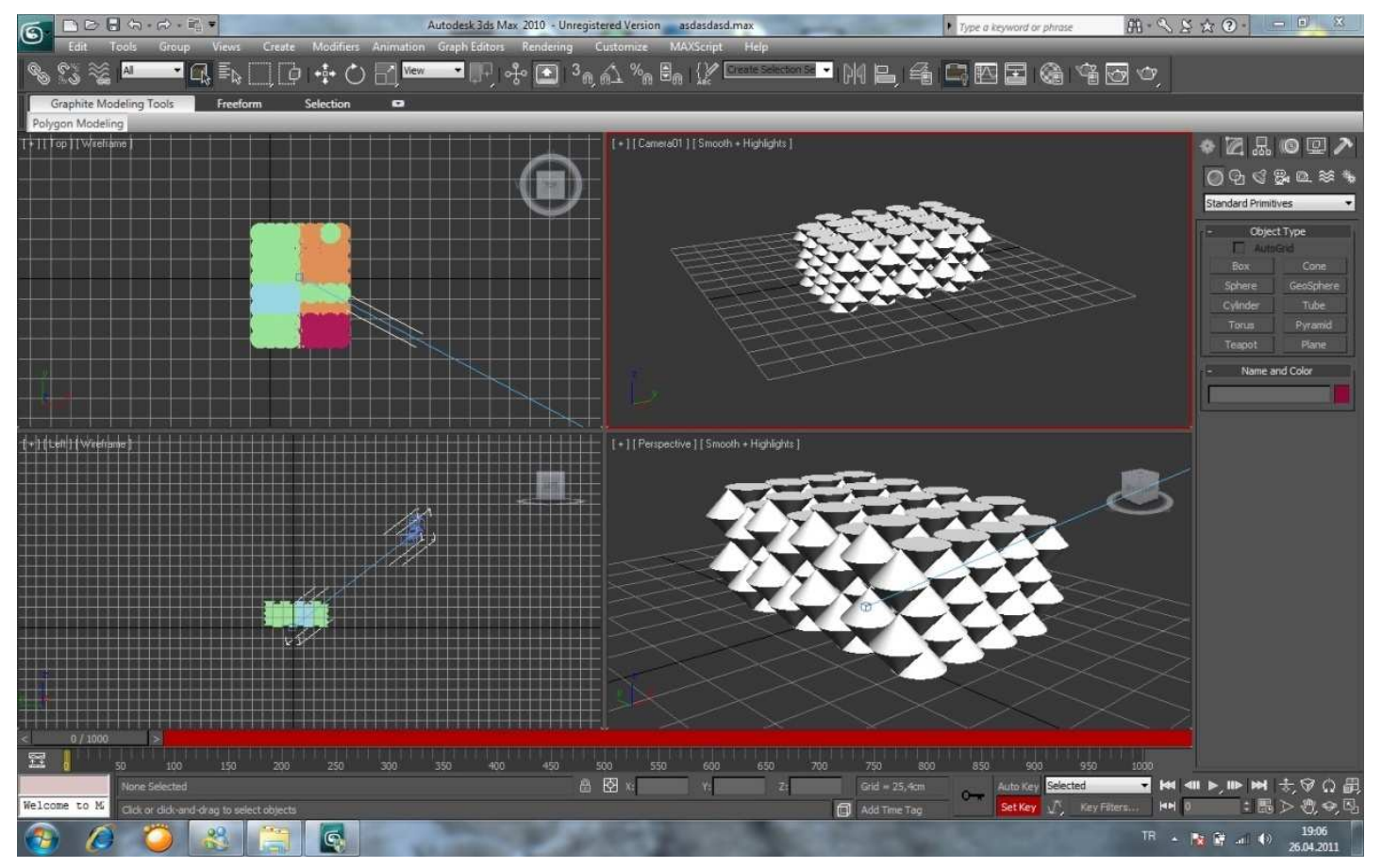

Figure A.5: Snapshot of a video file at the beginning

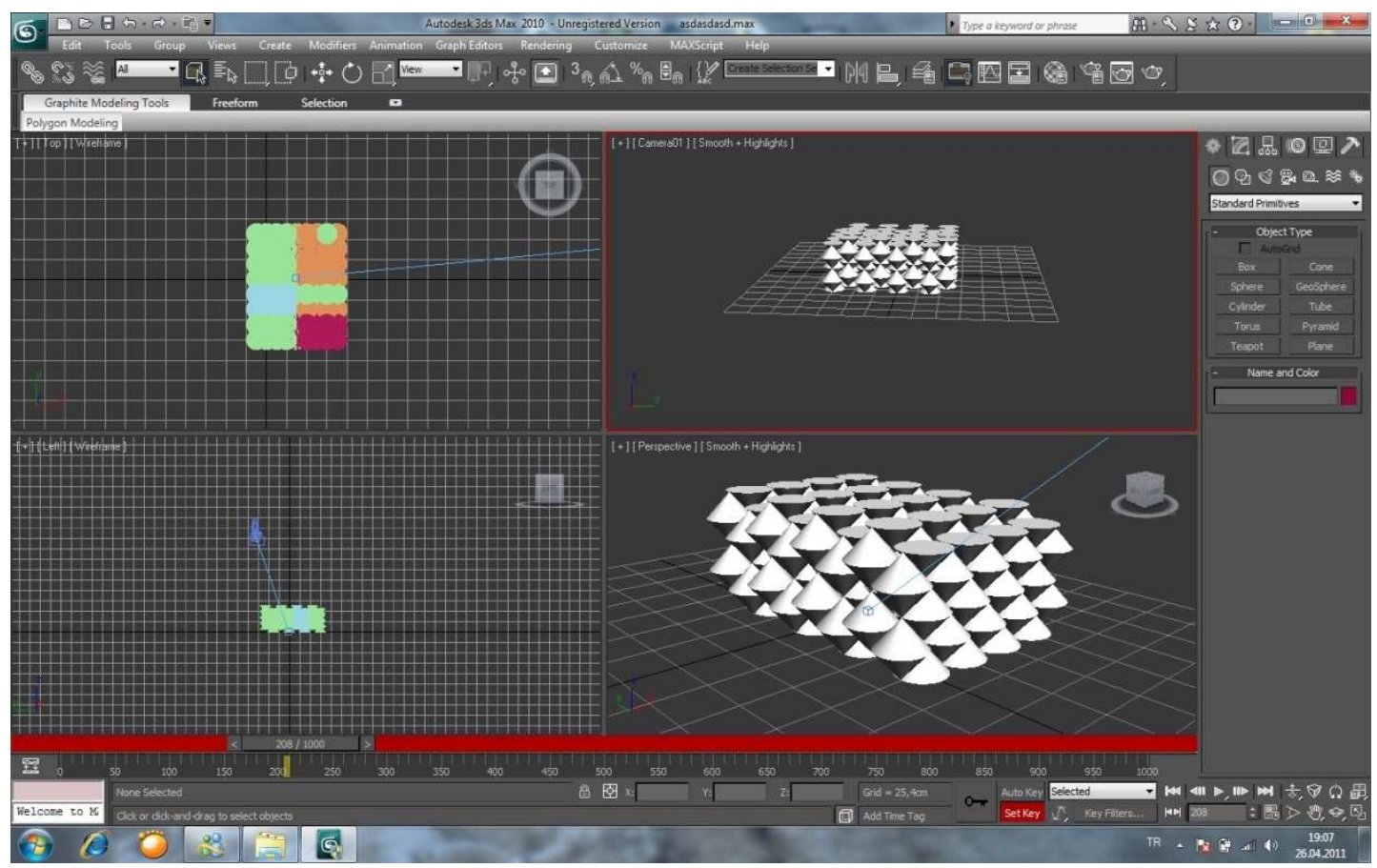

Figure A.6: Snapshot of a video file at 5 secs 


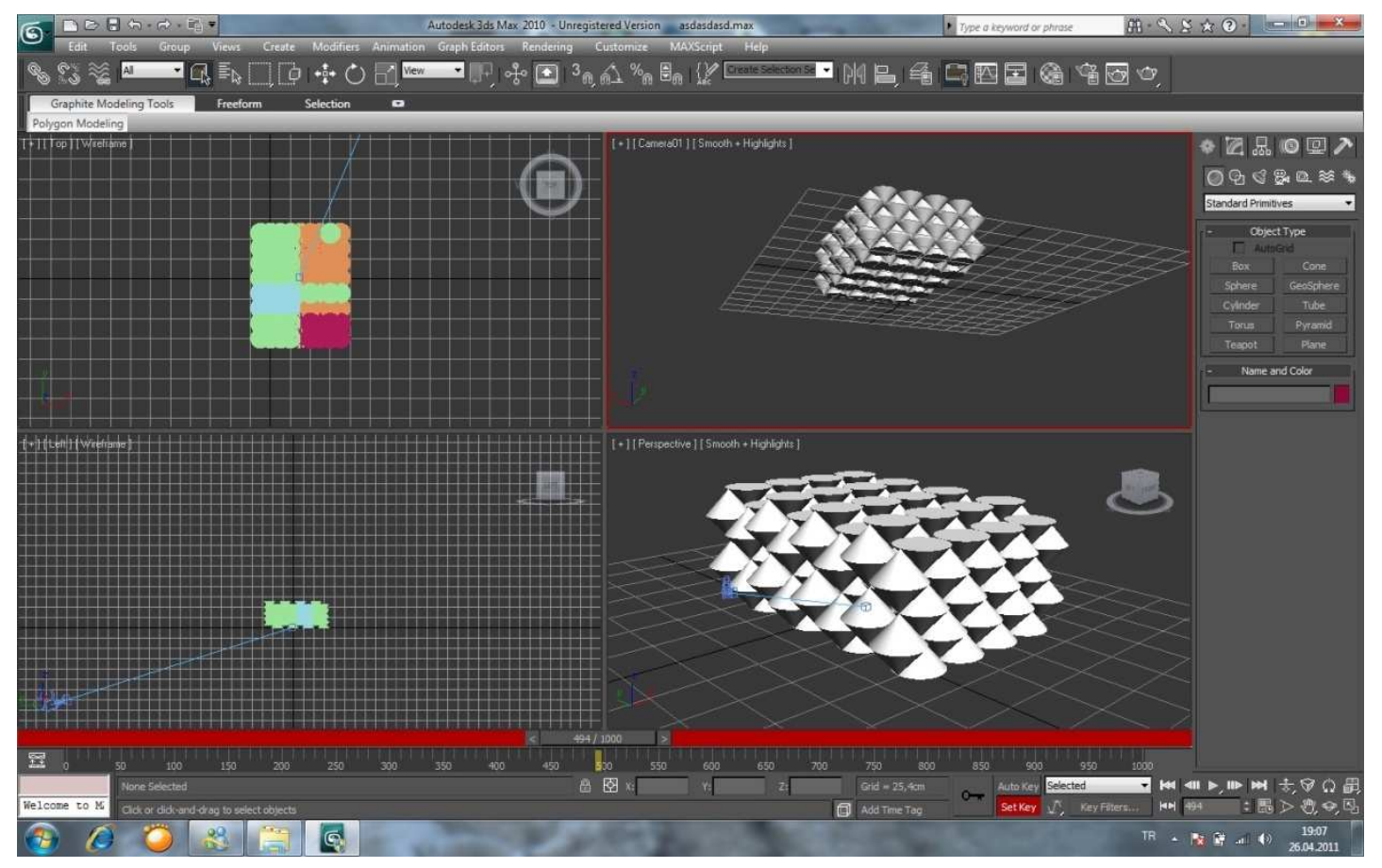

Figure A.7: Snapshot of a video file at $15 \mathrm{secs}$

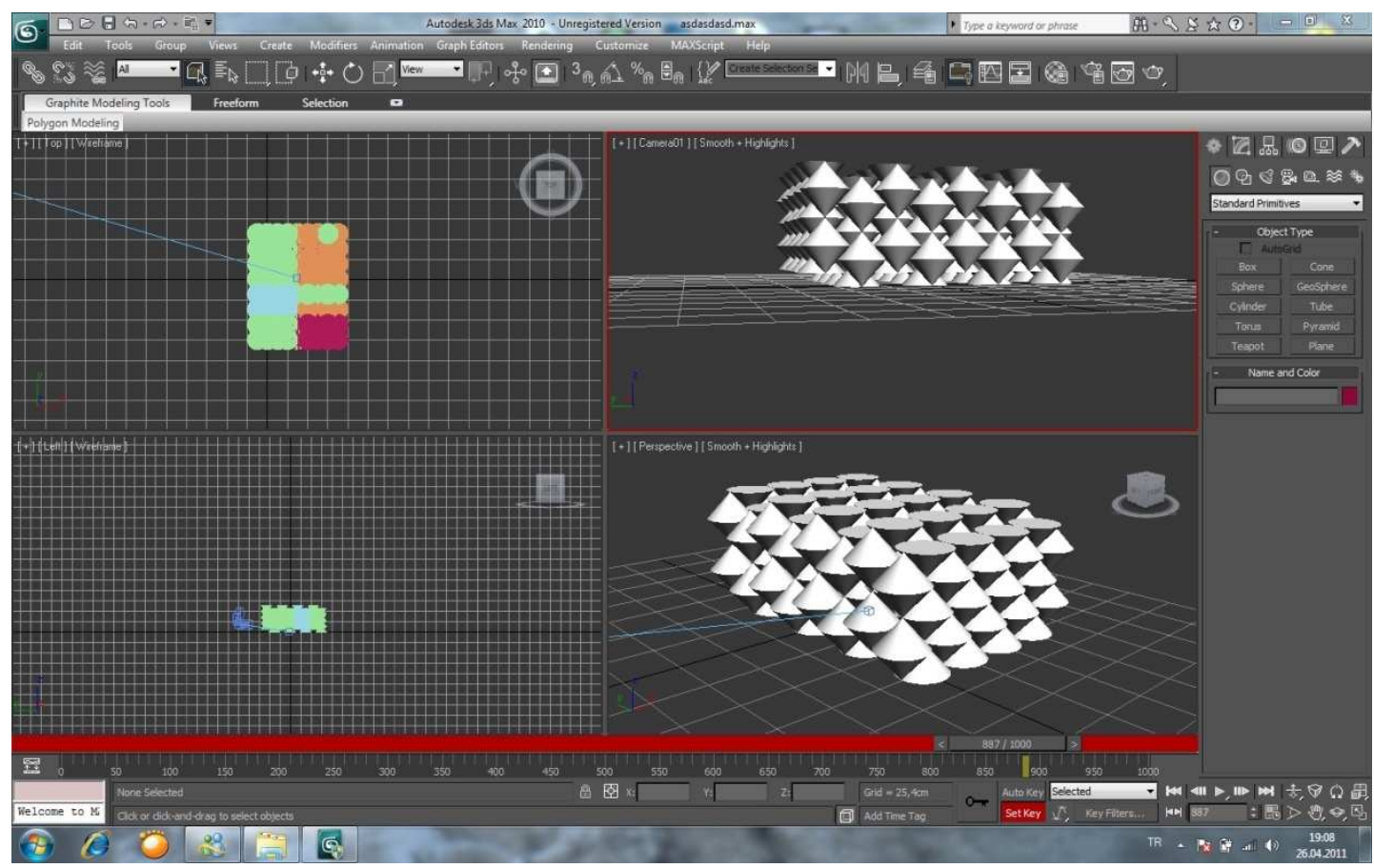

Figure A.8: Snapshot of a video file at $25 \mathrm{secs}$ 


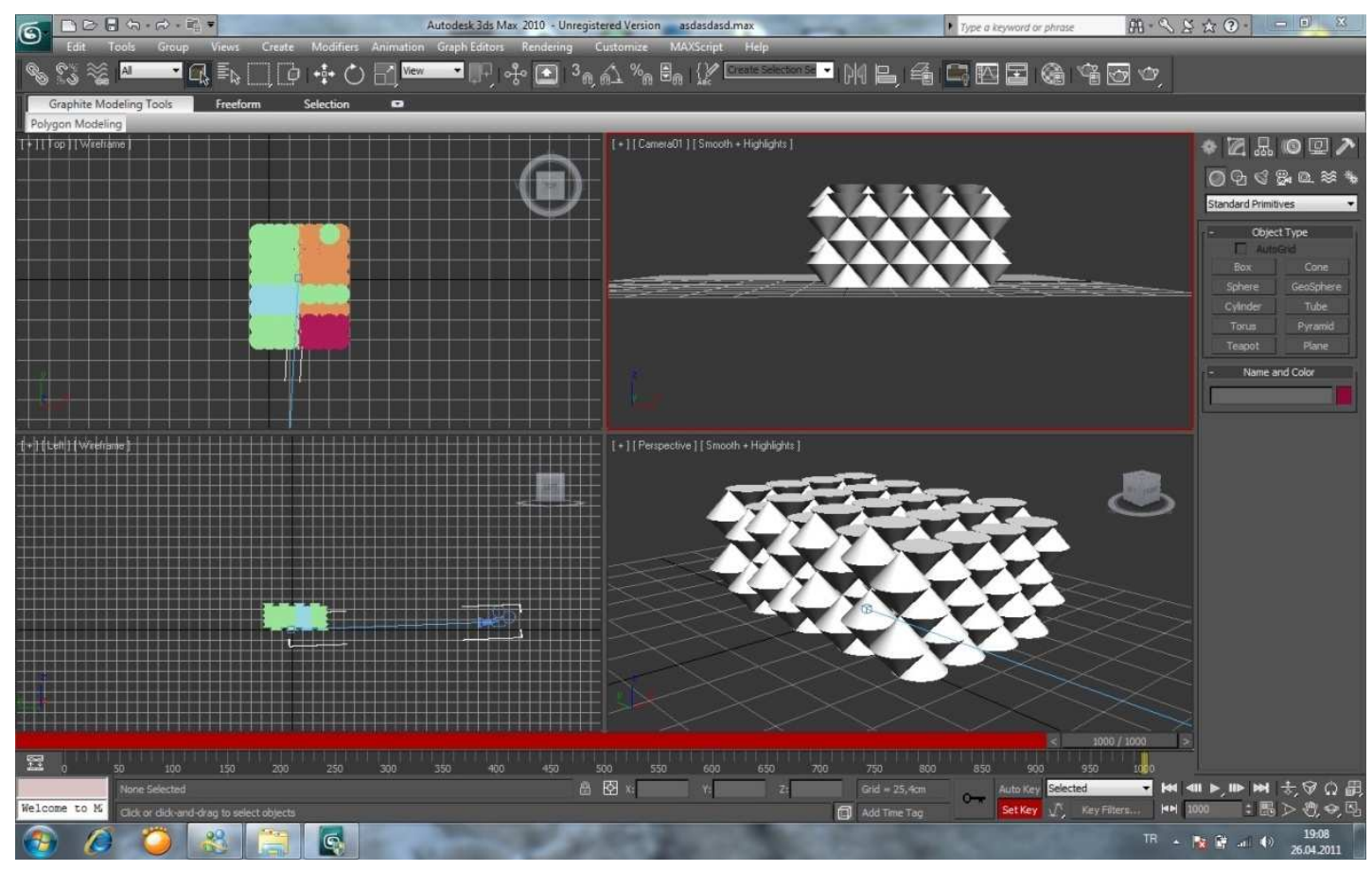

Figure A.9: Snapshot of a video file at the end

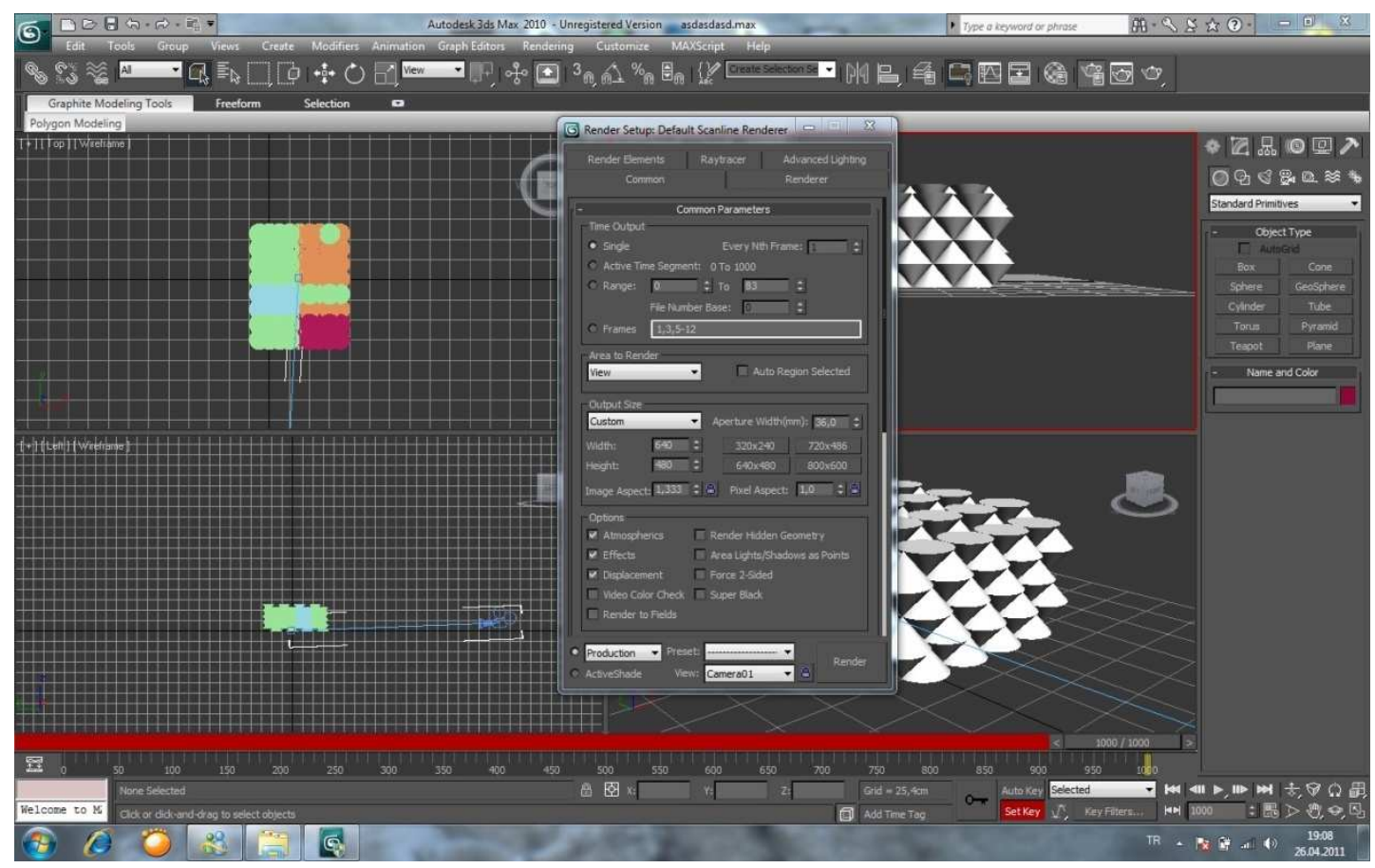

Figure A.10: Initializing video properties 


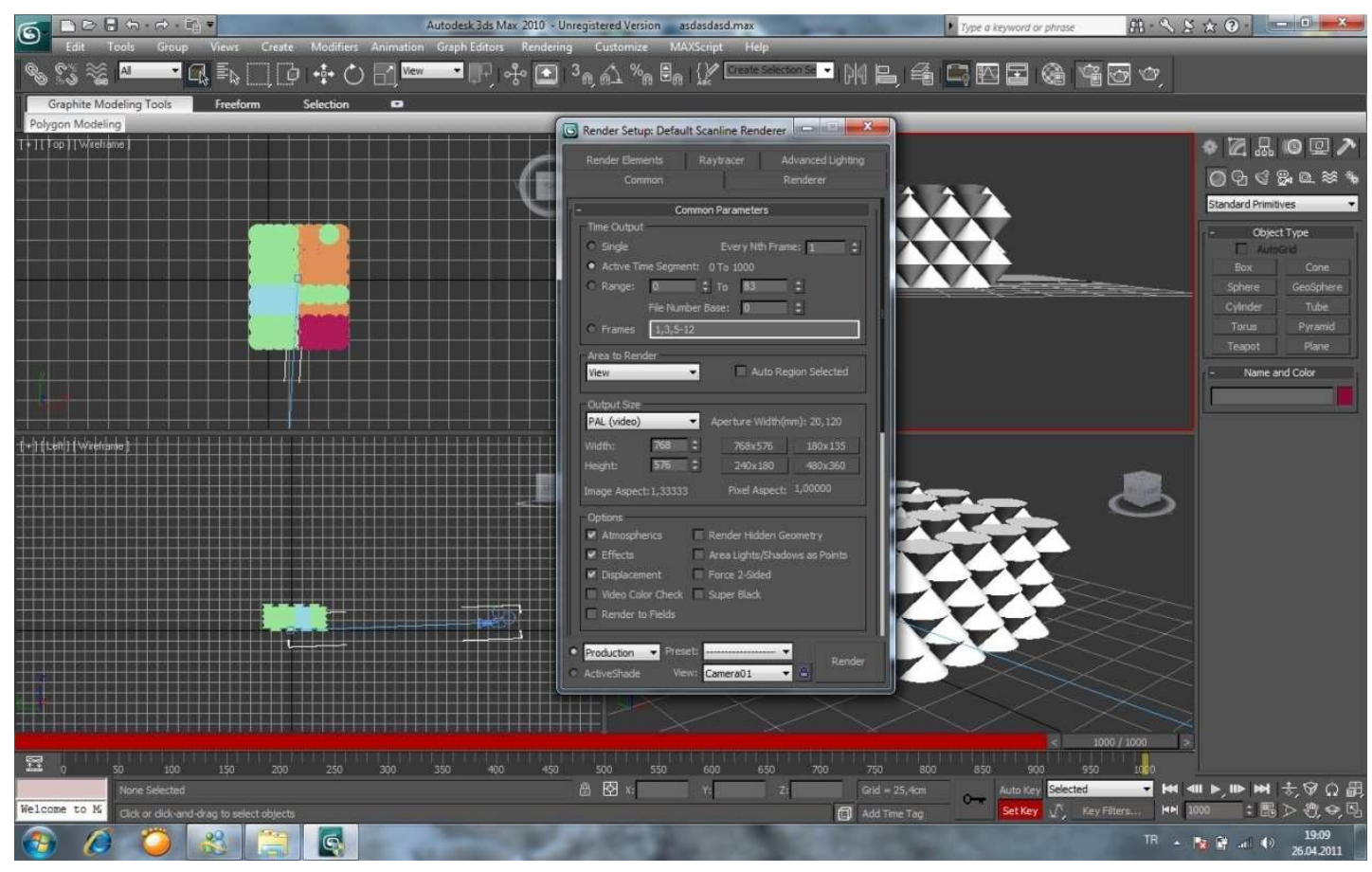

Figure A.11: Render setup for completing the video

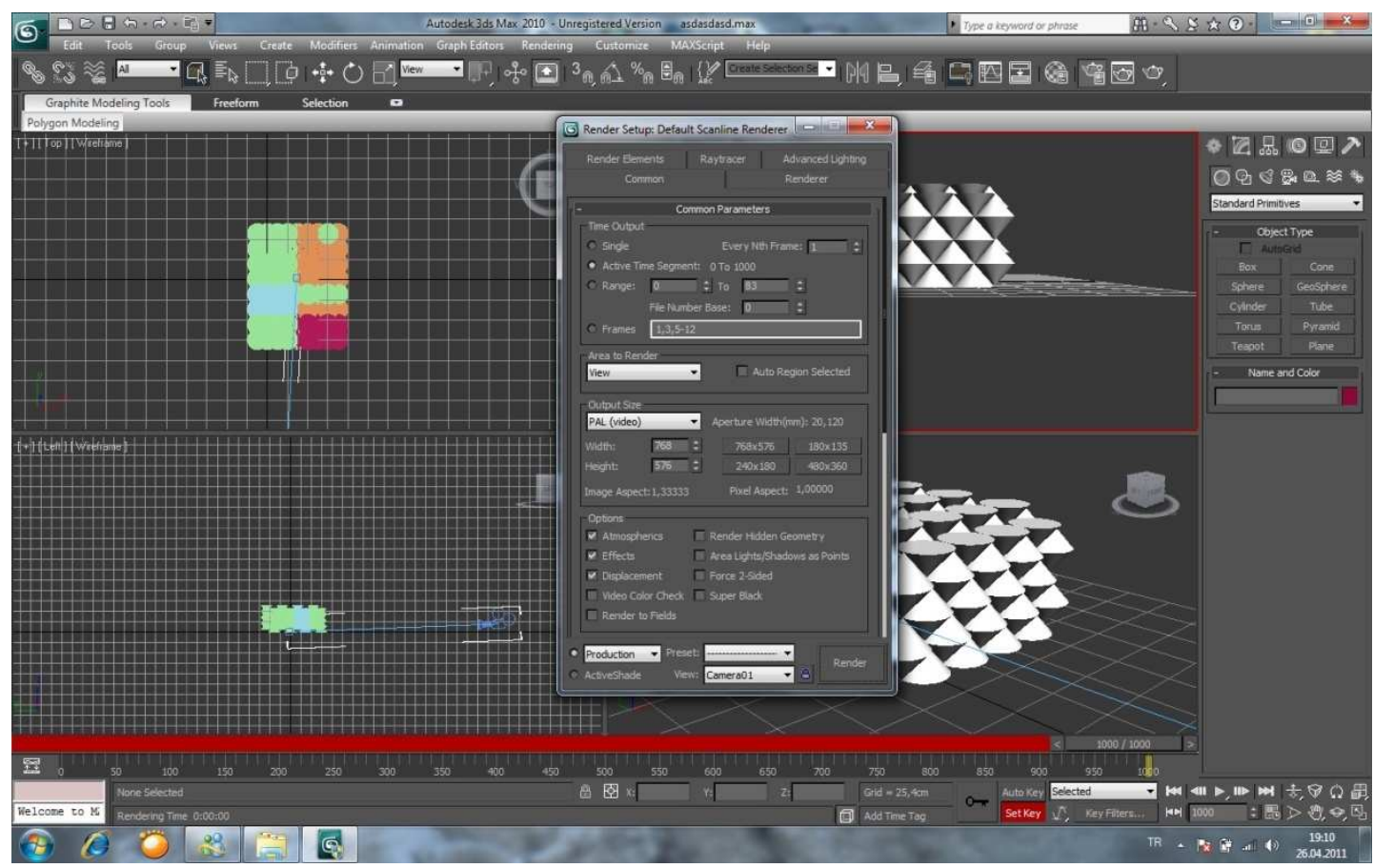

Figure A.12: Render setup for starting the video 


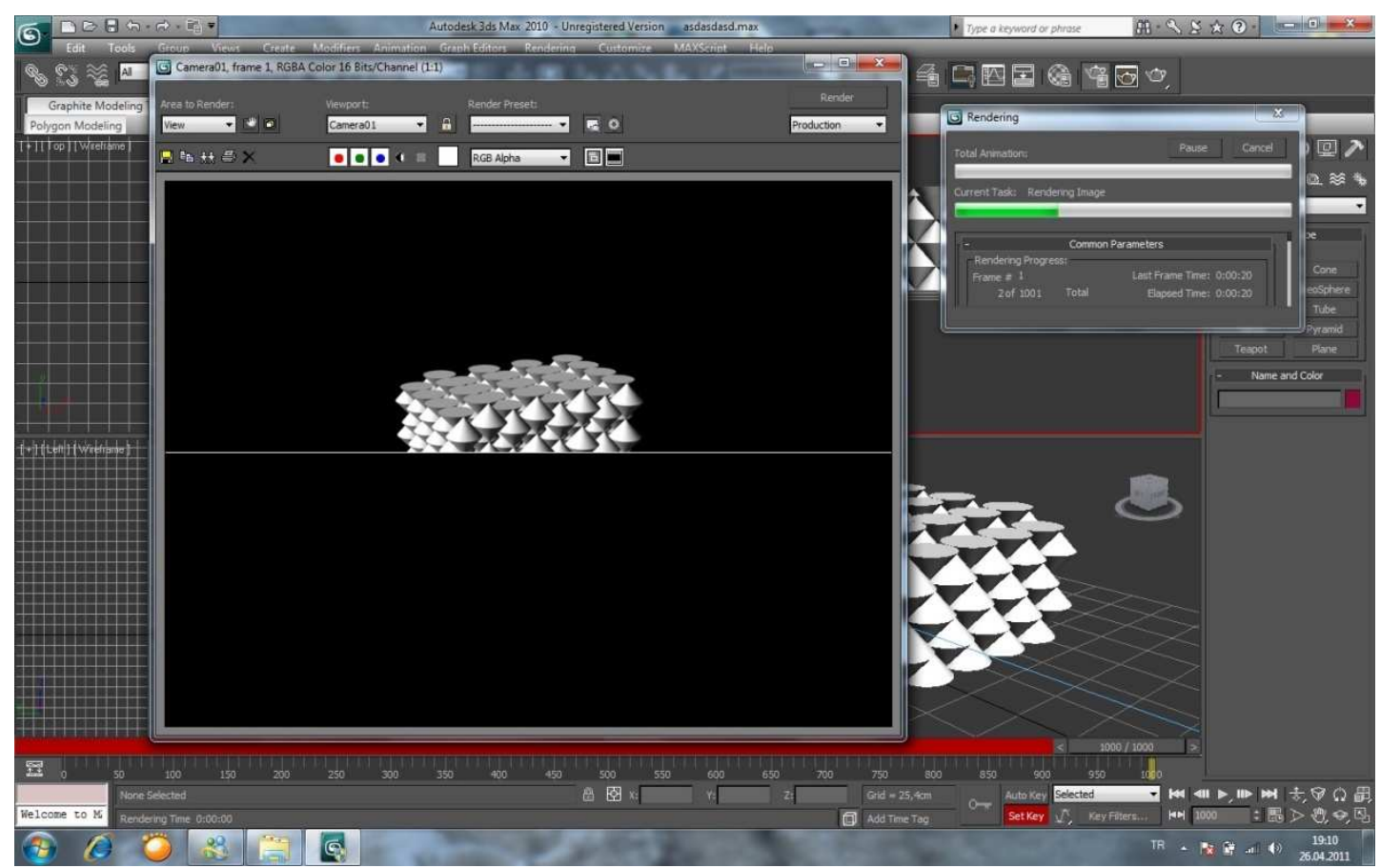

Figure A.13: Snapshot of a video while it started 